\title{
Sharp weak type estimates for weights in the class $A_{p_{1}, p_{2}}$
}

\author{
Alexander Reznikov
}

\begin{abstract}
We get sharp estimates for the distribution function of nonnegative weights that satisfy the so-called $A_{p_{1}, p_{2}}$ condition. For particular choices of parameters $p_{1}, p_{2}$ this condition becomes an $A_{p}$-condition or reverse Hölder condition. We also get maximizers for these sharp estimates. We use the Bellman technique and try to carefully present and motivate our tactics. As an illustration of how these results can be used, we deduce the following result: if a weight $w$ is in $A_{2}$ then it self-improves to a weight that satisfies a reverse Hölder condition.
\end{abstract}

\section{Introduction}

\subsection{Problem setting: basic definitions}

Put $I=[0,1]$ and take $p_{1}>p_{2}, p_{i} \neq 0, \pm \infty$. For every nonnegative function $\varphi$ and any interval $J \subset I$ we denote

$$
\langle\varphi\rangle_{J}=\frac{1}{|J|} \int_{J} \varphi(t) d t,
$$

where $|J|$ is the length of the interval $J$. For simplicity, when we take an average over the whole interval $I$, we drop the subindex and write $\langle\varphi\rangle$.

Take a nonnegative function $w$. Note that by the Hölder inequality we have

$$
\left\langle w^{p_{1}}\right\rangle_{J}^{1 / p_{1}} \geqslant\left\langle w^{p_{2}}\right\rangle_{J}^{1 / p_{2}} .
$$

Let $Q>1$. We are going to consider such functions $w \geqslant 0$ that the following "reverse" inequality is true:

$$
\left\langle w^{p_{1}}\right\rangle_{J}^{1 / p_{1}} \leqslant Q\left\langle w^{p_{2}}\right\rangle_{J}^{1 / p_{2}} \quad \forall J \subset I .
$$

If $p_{1}>p_{2}>1$ then (1.2) is called the reverse Hölder inequality. If $p_{1}=1$ and $p_{2}=-1 /(p-1)$ for a certain $p>1$, then (1.2) is the famous $A_{p}$-condition.

Mathematics Subject Classification (2010): 42B20, 42B25.

Keywords: Bellman function, $A_{p_{1}, p_{2}}$ weight, $A_{p}$ weight, Muckenhoupt weight, $R H_{p}$ weight, reverse Hölder condition. 
If $w \geqslant 0$ satisfies $(1.2)$, we write

$$
w \in A_{p_{1}, p_{2}}^{Q} .
$$

We are interested in the following question: how big can $w$ be? That is, for given $\lambda$, we want to estimate the measure of the set $\{t \in I: w(t) \geqslant \lambda\}$.

\subsection{Bellman setting and initial properties}

History of the question. $A_{p}$ weights play a key role in theory of singular integrals on weighted spaces, which has recently experienced rapid progress. The so-called $A_{2}, A_{2}-A_{\infty}$, and $A_{p}-A_{\infty}$ conjectures were proved in a short time period. Moreover, the $A_{1}-A_{\infty}$ theory was expanded; see, for example, [3]. In this theory the careful study of weights, in particular their self-improvement properties, was key.Our estimates allow to obtain such properties, as we show in Section 8. This is why we think that sharp estimates for distribution function of $A_{p_{1}, p_{2}}$ weights are interesting.

Bellman functions related to harmonic analysis appeared in the work of Burkholder, [1]. After that the first appearance was in the preprint of the paper by Nazarov, Treil and Volberg, [4]. Different methods to find an exact Bellman function were developed. The reader can find them in [6], [7], [11], [8], and [9].

There are two works by V. Vasyunin, [8] and [9], which are related to the question with which we are concerned. He gave a sharp estimate of $\left\langle w^{q}\right\rangle$ for every $q \in \mathbb{R}$, with the assumption that $w \in A_{p_{1}, p_{2}}$. After the work [8], M. Dindoš and T. Wall, [2], found the sharp $A_{p^{-}}$"norm" of a function in a reverse Hölder class. V. Vasyunin used a Bellman approach and we shall follow it. However, we should make some changes, since in Vasyunin's work he was able to reduce the question to solving a certain ODE. We cannot do this, and we are going to solve a PDE, following the Monge-Ampère technique, see [11].

An important precursor for our study was the work of Vasyunin [10] on the weak-type John-Nirenberg inequality. The preprint [10] does not contain proofs, but the explicit Bellman functions stated there were found as solutions of the Monge-Ampère equation on a nonconvex plane domain, just as they are here. The function classes under consideration, the Bellman domains, the Monge-Ampère geometry, and the optimizers in the two cases are different; nonetheless, the basic steps in the constructions and the proofs are the same.

Finally, we mention that applications of the estimates we are giving arise in many questions related to Calderón-Zygmund operators. In Section 8 we show how they can be used to deduce a sharp reverse Hölder inequality for $A_{2}$ weights. Such inequalities are very useful; we refer the reader to [5] and [3].

Acknowledgements. I am infinitely grateful to Professors Vasily Vasyunin and Alexander Volberg for spending a lot of time discussing this problem with me. I thank the referee for all his suggestions, which made this paper a lot easier to read. I also want to thank Carlos Pérez and the organizers of the 19th Summer St. Petersburg Meeting in Mathematical Analysis and the Analysis and PDE seminar 
at Michigan State University for an opportunity to present these results. Finally and most importantly, I want to thank my mother for all her support.

Initial definitions. Denote

$$
\Omega=\left\{x=\left(x_{1}, x_{2}\right): x_{i} \geqslant 0, x_{2}^{1 / p_{2}} \leqslant x_{1}^{1 / p_{1}} \leqslant Q x_{2}^{1 / p_{2}}\right\} .
$$

For every point $x \in \Omega$ we set

$$
\mathcal{B}\left(x_{1}, x_{2} ; \lambda\right)=\sup \left\{|\{t: w(t) \geqslant \lambda\}|:\left\langle w^{p_{1}}\right\rangle=x_{1},\left\langle w^{p_{2}}\right\rangle=x_{2}, w \in A_{p_{1}, p_{2}}^{Q}\right\} .
$$

Note that the definition of the $\Omega$ is due to inequalities (1.1) and (1.2).

We also need the following remark:

Remark 1.1. For every point $x=\left(x_{1}, x_{2}\right) \in \Omega$ there is a function $w \in A_{p_{1}, p_{2}}^{Q}$ such that $\left\langle w^{p_{1}}\right\rangle=x_{1}$ and $\left\langle w^{p_{2}}\right\rangle=x_{2}$.

The proof of this statement is subsumed in the proof of Lemma 2.7. This remark shows that $\mathcal{B}$ is defined (not equal to $-\infty$ ) on the whole domain $\Omega$.

Remark 1.2. Obviously, if $\lambda \leqslant 0$ then $\mathcal{B}(x ; \lambda)=1$ for every $x$. In the future we consider only $\lambda>0$.

For all $Q \geqslant 1$ define

$$
\Gamma_{Q}=\left\{\left(x_{1}, x_{2}\right): x_{i} \geqslant 0, x_{1}^{1 / p_{1}}=Q x_{2}^{1 / p_{2}}\right\} .
$$

Lemma 1.3. Let $\left(v^{p_{1}}, v^{p_{2}}\right) \in \Gamma_{1}$. Then

$$
\mathcal{B}\left(v^{p_{1}}, v^{p_{2}} ; \lambda\right)= \begin{cases}1, & v \geqslant \lambda, \\ 0, & v<\lambda .\end{cases}
$$

Proof. Let $\left\langle w^{p_{1}}\right\rangle=v^{p_{1}},\left\langle w^{p_{2}}\right\rangle=v^{p_{2}}$. Then Hölder's inequality becomes an equality and therefore $w$ is identically equal to $v$. Thus if $v \geqslant \lambda$ then $\{t: w(t) \geqslant \lambda\}=I$ and $\mathcal{B}\left(v^{p_{1}}, v^{p_{2}} ; \lambda\right)=1$. Similarly, if $v<\lambda$ then $\mathcal{B}\left(v^{p_{1}}, v^{p_{2}} ; \lambda\right)=0$.

Now we are going to get rid of the $\lambda$ using homogeneity. Take $w \in A_{p_{1}, p_{2}}^{Q}$ with $\left\langle w^{p_{1}}\right\rangle=x_{1}$ and $\left\langle w^{p_{2}}\right\rangle=x_{2}$. For a positive number $s$ write $\tilde{w}(t)=s w(t)$. Then $\tilde{w} \in A_{p_{1}, p_{2}}^{Q},\left\langle\tilde{w}^{p_{1}}\right\rangle=s^{p_{1}} x_{1}$ and $\left\langle\tilde{w}^{p_{2}}\right\rangle=s^{p_{2}} x_{2}$. Also

$$
w(t) \geqslant \lambda \Longleftrightarrow \tilde{w}(t) \geqslant s \lambda \text {. }
$$

Therefore,

$$
\mathcal{B}\left(x_{1}, x_{2} ; \lambda\right)=\mathcal{B}\left(s^{p_{1}} x_{1}, s^{p_{2}} x_{2} ; s \lambda\right) .
$$

Put $s=1 / \lambda$. Then we get

$$
\mathcal{B}\left(x_{1}, x_{2} ; \lambda\right)=\mathcal{B}\left(\lambda^{-p_{1}} x_{1}, \lambda^{-p_{2}} x_{2} ; 1\right),
$$

so it suffices to find only $\mathcal{B}\left(x_{1}, x_{2} ; 1\right)$ for every $\left(x_{1}, x_{2}\right) \in \Omega$. We set

$$
\mathcal{B}\left(x_{1}, x_{2}\right)=\mathcal{B}\left(x_{1}, x_{2} ; 1\right) .
$$


Lemma 1.3 says that

$$
\mathcal{B}\left(v^{p_{1}}, v^{p_{2}}\right)= \begin{cases}1, & v \geqslant 1 \\ 0, & v<1\end{cases}
$$

\subsection{Structure of the paper}

We describe the structure of the paper. The strategy is the following: we deduce some heuristic properties of function $\mathcal{B}$ and then find a candidate function $B$ with these properties. We then rigorously prove that $B=\mathcal{B}$.

In Subsections 1.4 and 1.5 we informally derive the main property of $B$, its degenerate local concavity. This property means that we should look for solutions of the homogeneous Monge-Ampère equation on $\Omega$; in Subsection 1.6 a useful theorem about such equations is stated. In Section 2 we make several technical calculations. Further, in Section 3 we find an appropriate candidate $B$ using the Monge-Ampère machinery of Subsection 1.6. The reader can read [11] for more examples.

After we find a function $B$ that is the most natural candidate for our Bellman function, we start proving that $B=\mathcal{B}$. In Section 4 we prove that $B \geqslant \mathcal{B}$, using induction on scales.

To prove that $B \leqslant \mathcal{B}$, we need to take an $x, x \in \Omega$, and find a function $w \in A_{p_{1}, p_{2}}^{Q}$ such that $\left(\left\langle w^{p_{1}}\right\rangle,\left\langle w^{p_{2}}\right\rangle\right)=x$ and $B(x)=|\{t: w(t) \geqslant 1\}|$. We shall do it in Section 6. We also emphasize that the function $\mathcal{B}$ and its properties somehow carry information about attainability of the supremum (i.e., whether $\sup =\max$ ) and about the maximizer.

\subsection{The main property: local concavity}

We give the following definition:

Definition 1.4. A function $F$ is called locally concave in a domain $\Omega$ if for every $x \in \Omega$ and for every convex neighborhood $U$ of $x$ such that $U \subset \Omega$, the following inequality holds:

$$
F(\mu x+(1-\mu) y) \geqslant \mu F(x)+(1-\mu) F(y), \quad \forall y \in U, \quad \forall \mu \in[0,1] .
$$

In this section we will use some heuristics to derive the main property of the Bellman function. We cannot prove this property a priori, but we will use it to get an appropriate candidate for $\mathcal{B}$.

Consider two points $y=\left(y_{1}, y_{2}\right) \in \Omega$ and $z=\left(z_{1}, z_{2}\right) \in \Omega$ and the line segment connecting $y$ and $z:[y, z]=\{\mu y+(1-\mu) z: \mu \in[0,1]\} \subset \Omega$. Assume that the supremum in (1.3) is attained on functions $w_{y}$ and $w_{z}$ respectively (we note that we do not know whether the supremum is attained or not; we assume it is attained for simplicity). For some $\mu \in(0,1)$ take $x=\mu y+(1-\mu) z$-a point on $[y, z]$. Define

$$
w(t)= \begin{cases}w_{y}\left(\frac{t}{\mu}\right), & t \in[0, \mu), \\ w_{z}\left(\frac{t}{1-\mu}\right), & t \in[\mu, 1] .\end{cases}
$$


Then

$$
\left\langle w^{p_{k}}\right\rangle=\int_{0}^{\mu} w_{y}^{p_{k}}\left(\frac{t}{\mu}\right) d t+\int_{\mu}^{1} w_{z}^{p_{k}}\left(\frac{t}{1-\mu}\right) d t=\mu y_{k}+(1-\mu) z_{k}=x_{k} .
$$

To be able to compare $\mathcal{B}(x)$ with $|\{t: w(t) \geqslant 1\}|$ we need one more thing, namely, $w \in A_{p_{1}, p_{2}}^{Q}$. However, we cannot prove this. Nevertheless, if $w \in A_{p_{1}, p_{2}}^{Q}$ then

$$
\begin{aligned}
\mathcal{B}(x) \geqslant|\{t: w(t) \geqslant 1\}| & =\mu\left|\left\{t: w_{y}(t) \geqslant 1\right\}\right|+(1-\mu)\left|\left\{t: w_{z}(t) \geqslant 1\right\}\right| \\
& =\mu \mathcal{B}(y)+(1-\mu) \mathcal{B}(z) .
\end{aligned}
$$

Even though the above does not prove the local concavity of $\mathcal{B}$, we will search for a locally concave candidate $B$.

\subsection{Degeneration of the Hessian}

Assume that we have a smooth function $B$. Then $B$ is locally concave if and only if

$$
\frac{d^{2} B}{d x^{2}}=\left(\begin{array}{ll}
B_{x_{1} x_{1}}^{\prime \prime} & B_{x_{1} x_{2}}^{\prime \prime} \\
B_{x_{2} x_{1}}^{\prime \prime} & B_{x_{2} x_{2}}^{\prime \prime}
\end{array}\right) \leqslant 0
$$

Moreover, we want to find the best concave function. "Best" means that $B$ must be as small as possible (since we want to estimate something from above). This gives us hope that the local concavity is "sharp", i.e., that $\frac{d^{2} B}{d x^{2}}$ degenerates (as a trivial example we mention that in the one variable case a straight line represents the smallest concave function with fixed boundary values). Namely we require that, for every point $x \in \Omega$ there be a direction $\vec{m}(x)$ along which $B$ is a linear function. This just means that

$$
\operatorname{det}\left(\frac{d^{2} B}{d x^{2}}\right)=0
$$

Accordingly, we will look for a Bellman candidate satisfying condition (1.6).

\subsection{On the Monge-Ampère PDE}

In this subsection we state the following known result; see [7].

Theorem 1.5. Let $B$ be a $C^{2}$ function defined in $\Omega$ and assume that either $B_{x_{1} x_{1}}^{\prime \prime} \neq 0$ or $B_{x_{2} x_{2}}^{\prime \prime} \neq 0$, and

$$
\operatorname{det}\left(\frac{d^{2} B}{d x^{2}}\right)=0
$$

Let

$$
t_{1}=B_{x_{1}}^{\prime}, \quad t_{2}=B_{x_{2}}^{\prime}, \quad t_{0}=B-x_{1} t_{1}-x_{2} t_{2} .
$$

Then the functions $t_{k}$ are constant on each integral trajectory generated by the kernel of the Hessian $\frac{d^{2} B}{d x^{2}}$. Moreover, these integral trajectories are straight lines given by

$$
d t_{0}+x_{1} d t_{1}+x_{2} d t_{2}=0
$$




\section{All technical calculations}

In this section we shall state and prove a number of formulas that we will need in the future.

\subsection{Initial calculations}

We start with formalizing the geometry of $\Omega$. Our first lemma is the following:

Lemma 2.1. For every $Q>1$, there are two solutions $\gamma_{ \pm}\left(0<\gamma_{-}<1<\gamma_{+}\right)$of the following equation:

$$
Q^{-p_{2}}\left(1-\frac{p_{2}}{p_{1}}\right) \gamma^{p_{2}}=1-\frac{p_{2}}{p_{1}} Q^{-p_{2}} \gamma^{p_{2}-p_{1}}
$$

Proof. Put

$$
f(t)=\left(1-\frac{p_{2}}{p_{1}}\right) t^{p_{2}}+\frac{p_{2}}{p_{1}} t^{p_{2}-p_{1}} .
$$

We want to prove that there are two values of $t$ such that $f(t)=Q^{p_{2}}$. Obviously,

$$
f^{\prime}(t)=p_{2} \frac{p_{1}-p_{2}}{p_{1}} t^{p_{2}-1}+\frac{p_{2}}{p_{1}}\left(p_{2}-p_{1}\right) t^{p_{2}-p_{1}-1}=\frac{p_{2}}{p_{1}}\left(p_{1}-p_{2}\right) t^{p_{2}-p_{1}-1}\left(t^{p_{1}}-1\right) .
$$

Observe that

$$
\operatorname{sign}\left(\frac{t^{p_{1}}-1}{p_{1}}\right)=\operatorname{sign}(t-1),
$$

So

$$
\operatorname{sign}\left(f^{\prime}(t)\right)=\operatorname{sign}\left(p_{2}(t-1)\right) .
$$

Now we consider two cases.

Case 1: $p_{2}>0$. Then $f(0)=\infty, f(\infty)=\infty$ and $f(1)=1$. Moreover, when $t \in[0,1], f(t)$ decreases from $\infty$ to 1 ; when $t \in[1, \infty], f(t)$ increases from 1 to $\infty$. The observation that $Q^{p_{2}}>1$ finishes the proof for this case.

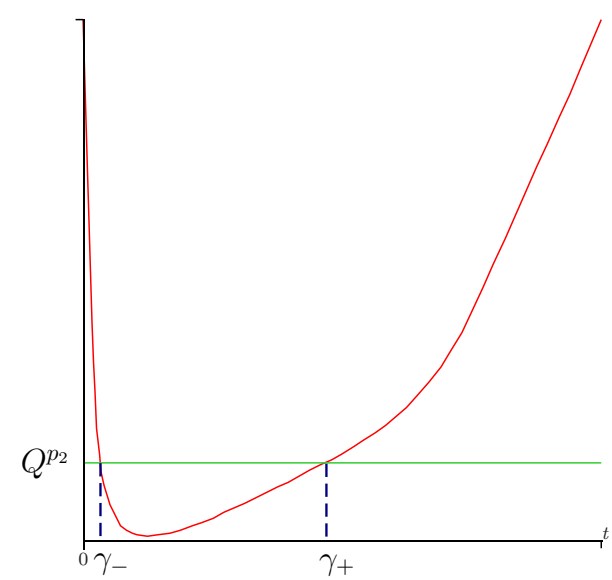

Figure 1: The function $f(t)$ for $p_{2}>0$. 


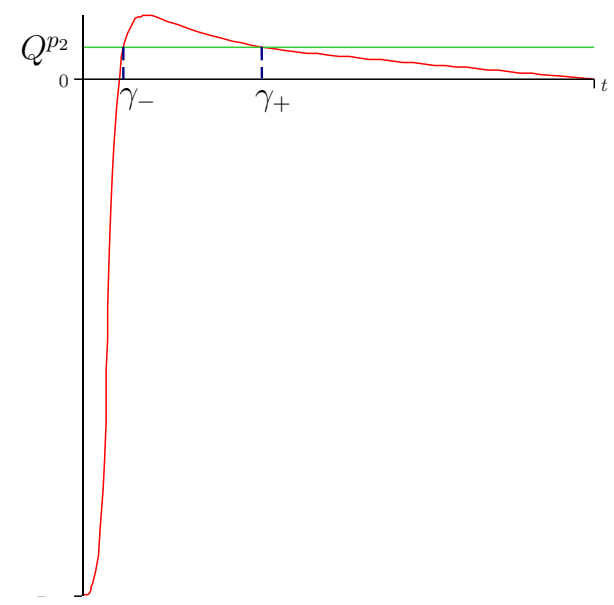

Figure 2: The function $f(t)$ for $p_{2}<0$.

Case 2: $p_{2}<0$. Then $f(0)=-\infty, f(\infty)=0$ and $f(1)=1$. Moreover, when $t \in[0,1], f(t)$ increases from $-\infty$ to 1 ; when $t \in[1, \infty], f(t)$ decreases from 1 to 0 . The observation that $Q^{p_{2}}<1$ finishes the proof.

Lemma 2.2. For every point $\left(v^{p_{1}}, v^{p_{2}}\right) \in \Gamma_{1}$ there are two tangent lines to $\Gamma_{Q}$, $\ell_{+}(v)$ and $\ell_{-}(v)$, such that $\left(v^{p_{1}}, v^{p_{2}}\right) \in \ell_{ \pm}(v)$. These tangent lines are defined by the equations

$$
x_{2}=\frac{p_{2}}{p_{1}} Q^{-p_{2}} a_{ \pm}^{p_{2}-p_{1}}\left(x_{1}-v^{p_{1}}\right)+v^{p_{2}}
$$

where $a_{ \pm}=\gamma_{ \pm} v$.

Proof. Let $\left(v^{p_{1}}, v^{p_{2}}\right) \in \Gamma_{1}$. Then

$$
\left(a_{ \pm}^{p_{1}}, Q^{-p_{2}} a_{ \pm}^{p_{2}}\right) \in \Gamma_{Q} .
$$

Let $\ell_{ \pm}(v)$ be the lines given by

$$
x_{2}=\frac{p_{2}}{p_{1}} Q^{-p_{2}} a_{ \pm}^{p_{2}-p_{1}}\left(x_{1}-v^{p_{1}}\right)+v^{p_{2}} .
$$

First of all, $\left(v^{p_{1}}, v^{p_{2}}\right) \in \ell_{ \pm}(v)$. Second,

$$
\left(a_{ \pm}^{p_{1}}, Q^{-p_{2}} a_{ \pm}^{p_{2}}\right) \in \ell_{ \pm}(v) .
$$

To prove this, we use the definition of $\gamma_{ \pm}$:

$$
Q^{-p_{2}}\left(1-\frac{p_{2}}{p_{1}}\right) \gamma_{ \pm}^{p_{2}}=1-\frac{p_{2}}{p_{1}} Q^{-p_{2}} \gamma_{ \pm}^{p_{2}-p_{1}}
$$

whence

$$
Q^{-p_{2}}\left(1-\frac{p_{2}}{p_{1}}\right) a_{ \pm}^{p_{2}}=v^{p_{2}}-\frac{p_{2}}{p_{1}} Q^{-p_{2}} a_{ \pm}^{p_{2}-p_{1}} v^{p_{1}}
$$


Therefore,

$$
Q^{-p_{2}} a_{ \pm}^{p_{2}}=\frac{p_{2}}{p_{1}} Q^{-p_{2}} a_{ \pm}^{p_{2}-p_{1}}\left(a_{ \pm}^{p_{1}}-v^{p_{1}}\right)+v^{p_{2}}
$$

which exactly means that

$$
\left(a_{ \pm}^{p_{1}}, Q^{-p_{2}} a_{ \pm}^{p_{2}}\right) \in \ell_{ \pm}(v)
$$

Also the slope of $\ell_{ \pm}(v)$ is equal to the derivative of the function $x_{2}=Q^{-p_{2}} x_{1}^{p_{2} / p_{1}}$ at the point $\left(a_{ \pm}^{p_{1}}, Q^{-p_{2}} a_{ \pm}^{p_{2}}\right)$, which finishes the proof.

Remark 2.3. If $p_{1}>0$, then $\gamma_{+}^{p_{1}}>1$ and we get $a_{+}^{p_{1}} / v^{p_{1}}>1$, so $a_{+}^{p_{1}}>v^{p_{1}}$. Thus, for every point $x=\left(x_{1}, x_{2}\right)$ on the segment of $\ell_{+}(v)$ with the endpoints $\left(v^{p_{1}}, v^{p_{2}}\right)$ and $\left(a_{+}^{p_{1}}, Q^{-p_{2}} a_{+}^{p_{2}}\right)$, we have $v^{p_{1}} \leqslant x_{1} \leqslant a_{+}^{p_{1}}$.

If $p_{1}<0$ then, for the same reason, $a_{+}^{p_{1}} \leqslant x_{1} \leqslant v^{p_{1}}$.

Corollary 2.4. Take a point $(1,1)$ and the corresponding tangents $\ell_{ \pm}=\ell_{ \pm}(1)$. They intersect $\Gamma_{1}$ one more time at the points $\left(v_{ \pm}^{p_{1}}, v_{ \pm}^{p_{2}}\right)$. These points are defined by the following equations:

$$
v_{-}=\frac{\gamma_{-}}{\gamma_{+}} \quad \text { and } \quad v_{+}=\frac{\gamma_{+}}{\gamma_{-}}
$$

This corollary is obvious: $\ell_{ \pm}$and $\ell_{\mp}\left(v_{ \pm}\right)$are the same lines, namely, these are the lines passing through the points $(1,1)$ and $\left(v_{ \pm}^{p_{1}}, v_{ \pm}^{p_{2}}\right)$ and tangent to $\Gamma_{Q}$ at $\left(\gamma_{ \pm}^{p_{1}}, Q^{-p_{2}} \gamma_{ \pm}^{p_{2}}\right)$

Lemma 2.5. Take $x=\left(x_{1}, x_{2}\right) \in \Omega, x \notin \Gamma_{Q}$. Then there are two lines tangent to $\Gamma_{Q}$ that pass through $x$.

The proof of this lemma is the same as the proof of Lemma 2.2. We need the following observation:

Lemma 2.6. The number $\gamma_{+}$defined above satisfies

$$
1-Q^{-p_{2}} \gamma_{+}^{p_{2}-p_{1}}>0
$$

Proof. Since

$$
Q^{-p_{2}}\left(1-\frac{p_{2}}{p_{1}}\right) \gamma_{ \pm}^{p_{2}}=1-\frac{p_{2}}{p_{1}} Q^{-p_{2}} \gamma_{ \pm}^{p_{2}-p_{1}}
$$

we get

$$
Q^{-p_{2}}\left(1-\frac{p_{2}}{p_{1}}\right) \gamma_{+}^{p_{2}}=1+\left(1-\frac{p_{2}}{p_{1}}\right) Q^{-p_{2}} \gamma_{+}^{p_{2}-p_{1}}-Q^{-p_{2}} \gamma_{+}^{p_{2}-p_{1}}
$$

so, using $\gamma_{+}>1$, we get

$$
1-Q^{-p_{2}} \gamma_{+}^{p_{2}-p_{1}}=\frac{p_{1}-p_{2}}{p_{1}} Q^{-p_{2}} \gamma_{+}^{p_{2}}\left(1-\gamma_{+}^{-p_{1}}\right)>0 .
$$


Our next lemma is the following observation about weights:

Lemma 2.7. Suppose we have two positive numbers $u$ and $v$ such that the line segment that connects the points $\left(u^{p_{1}}, u^{p_{2}}\right) \in \Gamma_{1}$ and $\left(v^{p_{1}}, v^{p_{2}}\right) \in \Gamma_{1}$ lies in $\Omega$. Suppose also that $\mu \in[0,1]$. Define

$$
w(t)= \begin{cases}u, & t \in[0, \mu), \\ v, & t \in[\mu, 1] .\end{cases}
$$

Then $w \in A_{p_{1}, p_{2}}^{Q}$.

Proof. We take an interval $J \subset I$. If $J \subset[0, \mu]$ or $J \subset[\mu, 1]$ then

$$
\left\langle w^{p_{1}}\right\rangle_{J}^{1 / p_{1}}\left\langle w^{p_{2}}\right\rangle_{J}^{-1 / p_{2}}=1<Q
$$

If $J=[\alpha, \beta], \alpha<\mu<\beta$ then

$$
\left\langle w^{p_{k}}\right\rangle_{J}=\frac{u^{p_{k}}(\mu-\alpha)+v^{p_{k}}(\beta-\mu)}{\beta-\alpha} .
$$

This means that the point $x=\left(\left\langle w^{p_{1}}\right\rangle_{J},\left\langle w^{p_{2}}\right\rangle_{J}\right)$ is a convex combination of the points $\left(u^{p_{1}}, u^{p_{2}}\right)$ and $\left(v^{p_{1}}, v^{p_{2}}\right)$, so $x \in \Omega$. Thus, $x_{1}^{1 / p_{1}} x_{2}^{-1 / p_{2}} \leqslant Q$, and, therefore, $w \in A_{p_{1}, p_{2}}^{Q}$.

Remark 2.8. In particular, if $J=I$, then we get that $\left\langle w^{p_{k}}\right\rangle=\mu u^{p_{k}}+(1-\mu) v^{p_{k}}$.

\subsection{Splitting of $\Omega$ : formulas}

Now we want to split $\Omega$ into different subdomains. We mention that our subdomains will be open, while $\Omega$ is closed. However, the reader will see that this detail is technical and will not affect our investigation. We write precise formulas and then explain their geometrical meaning using figures.

\section{Case 1. $p_{1}>p_{2}>0$}

$$
\begin{gathered}
\Omega_{\mathrm{I}}=\left\{x \in \Omega, x_{2}>Q^{-p_{2}} \frac{p_{2}}{p_{1}} \gamma_{+}^{p_{2}-p_{1}}\left(x_{1}-1\right)+1\right\} \cup\left\{x \in \Omega, x_{1}>\gamma_{+}^{p_{1}}\right\}, \\
\Omega_{\mathrm{II}}=\left\{x \in \Omega, x_{2}<Q^{-p_{2}} \frac{p_{2}}{p_{1}} \gamma_{+}^{p_{2}-p_{1}}\left(x_{1}-1\right)+1,\right. \\
\left.x_{2}<Q^{-p_{2}} \frac{p_{2}}{p_{1}} \gamma_{-}^{p_{2}-p_{1}}\left(x_{1}-1\right)+1, \gamma_{-}^{p_{1}}<x_{1}<\gamma_{+}^{p_{1}}\right\}, \\
\Omega_{\mathrm{III}}=\left\{x \in \Omega, x_{2}>Q^{-p_{2}} \frac{p_{2}}{p_{1}} \gamma_{-}^{p_{2}-p_{1}}\left(x_{1}-1\right)+1\right\}, \\
\Omega_{\mathrm{IV}}=\left\{x \in \Omega, x_{2}<Q^{-p_{2}} \frac{p_{2}}{p_{1}} \gamma_{-}^{p_{2}-p_{1}}\left(x_{1}-1\right)+1,0<x_{1}<\gamma_{-}^{p_{1}}\right\} .
\end{gathered}
$$




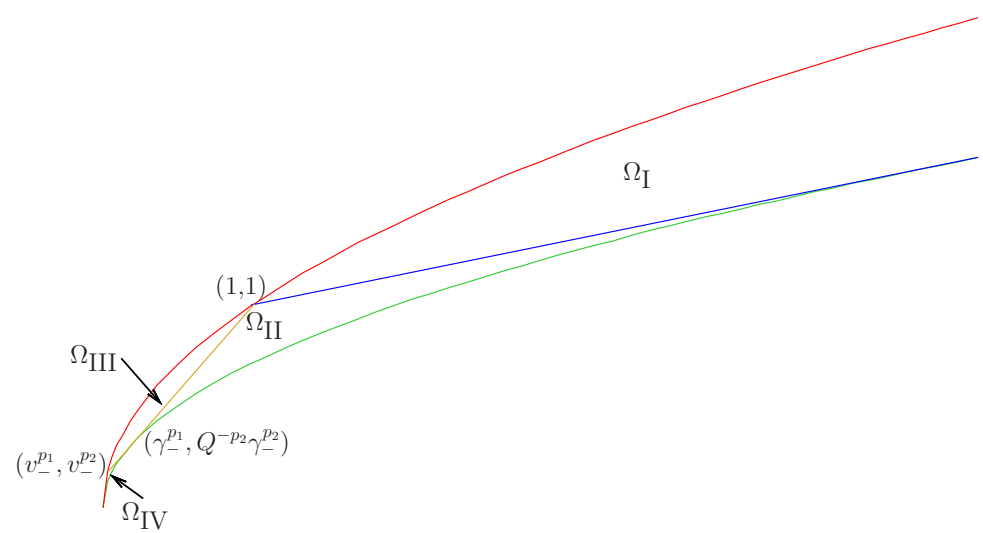

Figure 3: The domain $\Omega$ when $p_{1}>p_{2}>0$.

\section{Case 2. $p_{1}>0>p_{2}$}

$$
\begin{gathered}
\Omega_{\mathrm{I}}=\left\{x \in \Omega, x_{2}<Q^{-p_{2}} \frac{p_{2}}{p_{1}} \gamma_{+}^{p_{2}-p_{1}}\left(x_{1}-1\right)+1\right\} \cup\left\{x \in \Omega, x_{1}>\gamma_{+}^{p_{1}}\right\}, \\
\Omega_{\mathrm{II}}=\left\{x \in \Omega, x_{2}>Q^{-p_{2}} \frac{p_{2}}{p_{1}} \gamma_{+}^{p_{2}-p_{1}}\left(x_{1}-1\right)+1,\right. \\
\left.x_{2}>Q^{-p_{2}} \frac{p_{2}}{p_{1}} \gamma_{-}^{p_{2}-p_{1}}\left(x_{1}-1\right)+1, \gamma_{-}^{p_{1}}<x_{1}<\gamma_{+}^{p_{1}}\right\}, \\
\Omega_{\mathrm{III}}=\left\{x \in \Omega, x_{2}<Q^{-p_{2}} \frac{p_{2}}{p_{1}} \gamma_{-}^{p_{2}-p_{1}}\left(x_{1}-1\right)+1\right\}, \\
\Omega_{\mathrm{IV}}=\left\{x \in \Omega, x_{2}>Q^{-p_{2}} \frac{p_{2}}{p_{1}} \gamma_{-}^{p_{2}-p_{1}}\left(x_{1}-1\right)+1,0<x_{1}<\gamma_{-}^{p_{1}}\right\} .
\end{gathered}
$$

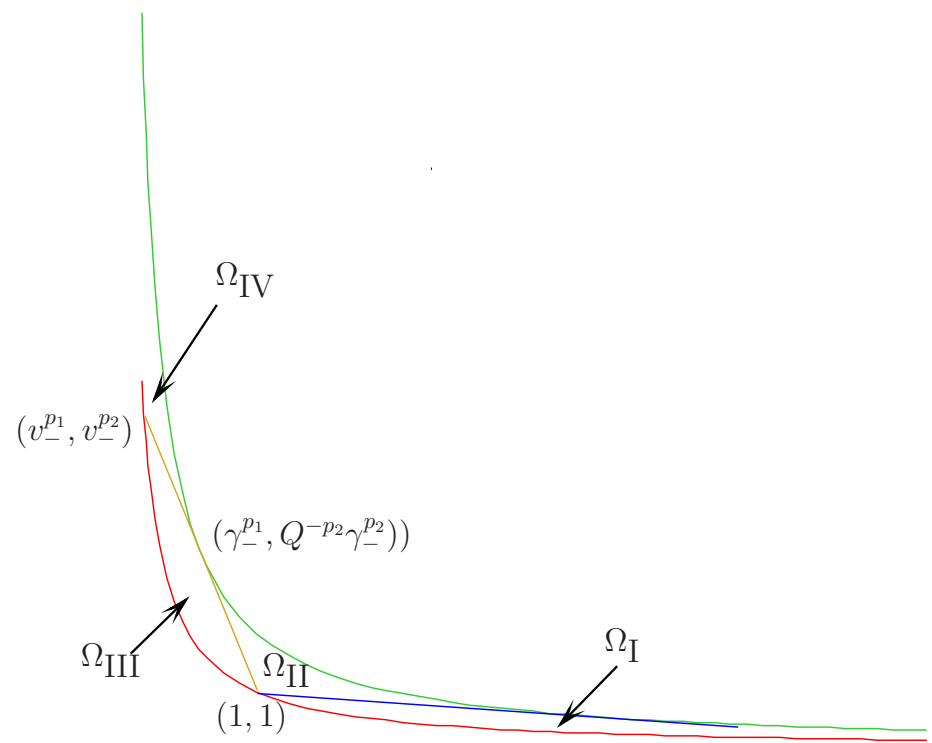

Figure 4: The domain $\Omega$ when $p_{1}>0>p_{2}$. 
Case 3. $0>p_{1}>p_{2}$

$$
\begin{aligned}
& \Omega_{\mathrm{I}}=\left\{x \in \Omega, x_{2}<Q^{-p_{2}} \frac{p_{2}}{p_{1}} \gamma_{+}^{p_{2}-p_{1}}\left(x_{1}-1\right)+1\right\} \cup\left\{x \in \Omega, x_{1}<\gamma_{+}^{p_{1}}\right\} \\
& \Omega_{\mathrm{II}}=\left\{x \in \Omega, x_{2}>Q^{-p_{2}} \frac{p_{2}}{p_{1}} \gamma_{+}^{p_{2}-p_{1}}\left(x_{1}-1\right)+1,\right. \\
& \left.x_{2}>Q^{-p_{2}} \frac{p_{2}}{p_{1}} \gamma_{-}^{p_{2}-p_{1}}\left(x_{1}-1\right)+1, \gamma_{+}^{p_{1}}<x_{1}<\gamma_{-}^{p_{1}}\right\}, \\
& \Omega_{\mathrm{III}}=\left\{x \in \Omega, x_{2}<Q^{-p_{2}} \frac{p_{2}}{p_{1}} \gamma_{-}^{p_{2}-p_{1}}\left(x_{1}-1\right)+1\right\}, \\
& \Omega_{\mathrm{IV}}=\left\{x \in x_{2}>Q^{-p_{2}} \frac{p_{2}}{p_{1}} \gamma_{-}^{p_{2}-p_{1}}\left(x_{1}-1\right)+1, x_{1}>\gamma_{-}^{p_{1}}\right\} .
\end{aligned}
$$

Figure 5: The domain $\Omega$ when $0>p_{1}>p_{2}$.

2.2.1. Motivation. We shall try to motivate why we chose these subdomains.

The reason we chose this splitting of $\Omega$ is that we can readily determine the Bellman function $B$ for some of the subdomains, namely $\Omega_{\mathrm{I}}$ and $\Omega_{\mathrm{III}}$. Let us explain. For each $\left(v^{p_{1}}, v^{p_{2}}\right) \in \Gamma_{1}$, the set of test functions over which the supremum in the definition of $\mathcal{B}$ is taken consists of a single constant function, $w_{\left(v^{p_{1}}, v^{p_{2}}\right)}=v$. Take two points $y, z \in\left\{\left(v^{p_{1}}, v^{p_{2}}\right): v>1\right\}$, such that the line segment $[y, z]$ lies entirely in $\Omega$, and any point $x$ on $[y, z]$. We can obtain a test function $w_{x}$ for $x$ simply by concatenating the two constant functions $w_{y}$ and $w_{z}$, as was done in Section 1.4 (by Lemma 2.7, this concatenation is in $A_{p_{1}, p_{2}}^{Q}$ ). Then $w_{x}$ is pointwise at least 1 , which means that $\mathcal{B}(x)=1$. Observe that by our definition $\Omega_{I}$ is precisely the set of all points $\mathrm{x}$ that can be obtained in this way.

We again emphasize that our intentions were to connect $x$ with two points $y$ and $z$ on the boundary $\Gamma_{1}$ that have pointwise big test-functions $w_{y}$ and $w_{z}$. Let us call these $y$ and $z$ good points. 
Take now $\Omega_{\text {III }}$. Here we cannot put $x$ on a line which connects two good points. However, we can connect $x$ with the point $(1,1)$, which is good, and with another point on $\Gamma$. This point is not good, but our test-function $w_{x}$, obtained as above, will be pointwise big enough due to the contribution of $w_{(1,1)}$. That is why we separate out this domain. We give the full details in Section 6.3.

Now we are left with $\Omega_{\mathrm{II}}$ and $\Omega_{\mathrm{IV}}$, and we do not subdivide them further.

\subsection{On the dependence of $v$ on $x$}

In this section we introduce a certain function $v$ as a function of $x$. We study its properties that will be important later. Here is the definition.

If $x \in \Omega_{\text {III }}$ we take $v \neq 1$ to be the solution of the following equation:

$$
v^{p_{2}}\left(1-x_{1}\right)-v^{p_{1}}\left(1-x_{2}\right)=x_{2}-x_{1} .
$$

The geometrical meaning of this is as follows. We take the line segment that connects the point $x$ and the point $(1,1)$. We continue this line until it intersects $\Gamma_{1}$ a second time. The point of intersection is exactly $\left(v^{p_{1}}, v^{p_{2}}\right)$. Note that for this $v$ we have $\operatorname{sign}\left(x_{1}-v^{p_{1}}\right)=\operatorname{sign}\left(p_{1}\right)$.

Next, take a point $\left(v^{p_{1}}, v^{p_{2}}\right)$ on the boundary of $\Omega_{\mathrm{IV}}$ and the tangent line $\ell_{+}(v)$. Such tangent lines foliate all $\Omega_{\mathrm{IV}}$, and they do not intersect. Therefore, for every $x \in \Omega_{\mathrm{IV}}$ we can find exactly one such $\ell_{+}(v)$. Notice that $\operatorname{sign}\left(x_{1}-v^{p_{1}}\right)=\operatorname{sign}\left(p_{1}\right)$.

Take this $\ell_{+}(v)$ :

$$
x_{2}=Q^{-p_{2}} \frac{p_{2}}{p_{1}} \gamma_{+}^{p_{2}-p_{1}} v^{p_{2}-p_{1}}\left(x_{1}-v^{p_{1}}\right)+v^{p_{2}} .
$$

Fix $x_{2}$ and consider $v$ as a function of $x_{1}$. We would like to determine the sign of $v_{x_{1}}^{\prime}$.

Definition 2.9. Take $v(x)$ to be a solution of the equations

$$
\begin{cases}v^{p_{2}}\left(1-x_{1}\right)-v^{p_{1}}\left(1-x_{2}\right)=x_{2}-x_{1}, & x \in \Omega_{\mathrm{III}}, \\ x_{2}=Q^{-p_{2}} \frac{p_{2}}{p_{1}} \gamma_{+}^{p_{2}-p_{1}} v^{p_{2}-p_{1}}\left(x_{1}-v^{p_{1}}\right)+v^{p_{2}}, & x \in \Omega_{\mathrm{IV}},\end{cases}
$$

and such that $\operatorname{sign}\left(x_{1}-v^{p_{1}}\right)=\operatorname{sign}\left(p_{1}\right)$.

Our goal is the following lemma:

Lemma 2.10. For every $x$ the following is true:

$$
\operatorname{sign}\left(v_{x_{1}}^{\prime}\right)=-\operatorname{sign}\left(p_{1}\right) \quad \text { and } \quad \operatorname{sign}\left(v_{x_{2}}^{\prime}\right)=\operatorname{sign}\left(p_{2}\right) .
$$

We split the proof into two cases.

Case 1: $x \in \Omega_{\mathrm{IV}}$. In the following lemma we determine the signs of $v_{x_{1}}^{\prime}$ and $v_{x_{2}}^{\prime}$ for $x \in \Omega_{\mathrm{IV}}$. 
Lemma 2.11. Take $x \in \Omega_{\mathrm{IV}}$. We define

$$
A=Q^{-p_{2}} \gamma_{+}^{p_{2}-p_{1}}, \quad \Pi=\frac{A x_{1}}{v^{p_{1}+1}}-\frac{x_{2}}{v^{p_{2}+1}} .
$$

Then

$$
v_{x_{1}}^{\prime}=\frac{1}{p_{1} \Pi} \frac{A}{v^{p_{1}}}, \quad v_{x_{2}}^{\prime}=-\frac{1}{p_{2} \Pi} \frac{1}{v^{p_{2}}} .
$$

Moreover, we always have $\Pi<0$ and therefore

$$
\operatorname{sign}\left(v_{x_{1}}^{\prime}\right)=-\operatorname{sign}\left(p_{1}\right) \quad \text { and } \quad \operatorname{sign}\left(v_{x_{2}}^{\prime}\right)=\operatorname{sign}\left(p_{2}\right) .
$$

Proof. Using the definition of $A$, rewrite the equation for $v$ in the following form:

$$
x_{2}=\frac{p_{2}}{p_{1}} A v^{p_{2}-p_{1}}\left(x_{1}-v^{p_{1}}\right)+v^{p_{2}}=\frac{p_{2}}{p_{1}} A v^{p_{2}-p_{1}} x_{1}-\frac{p_{2}}{p_{1}} A v^{p_{2}}+v^{p_{2}} .
$$

Hence,

$$
\frac{x_{2}}{v^{p_{2}}}=\frac{p_{2}}{p_{1}} A \frac{x_{1}}{v^{p_{1}}}-\left(\frac{p_{2}}{p_{1}} A-1\right) .
$$

Now, taking the partial derivative $\partial / \partial x_{1}$,

$$
-p_{2} \frac{x_{2}}{v^{p_{2}+1}} v_{x_{1}}^{\prime}=\frac{p_{2}}{p_{1}} A\left(\frac{1}{v^{p_{1}}}-\frac{p_{1} x_{1}}{v^{p_{1}+1}} v_{x_{1}}^{\prime}\right),
$$

and therefore,

$$
v_{x_{1}}^{\prime}\left(\frac{A x_{1}}{v^{p_{1}+1}}-\frac{x_{2}}{v^{p_{2}+1}}\right)=\frac{A}{p_{1} v^{p_{1}}} .
$$

One can get the result for $v_{x_{2}}^{\prime}$ similarly. From the equation

$$
\frac{x_{2}}{v^{p_{2}}}=\frac{p_{2}}{p_{1}} A \frac{x_{1}}{v^{p_{1}}}-\left(\frac{p_{2}}{p_{1}} A-1\right) .
$$

we get that

$$
\frac{A x_{1}}{v^{p_{1}}}-\frac{x_{2}}{v^{p_{2}}}=\frac{A x_{1}}{v^{p_{1}}}\left(1-\frac{p_{2}}{p_{1}}\right)+\left(\frac{p_{2}}{p_{1}} A-1\right) .
$$

From (2.1) we see that

$$
1-\frac{p_{2}}{p_{1}} A=Q^{-p_{2}}\left(1-\frac{p_{2}}{p_{1}}\right) \gamma_{+}^{p_{2}},
$$

so

$$
\begin{aligned}
\frac{A x_{1}}{v^{p_{1}}}-\frac{x_{2}}{v^{p_{2}}} & =\left(1-\frac{p_{2}}{p_{1}}\right)\left(\frac{Q^{-p_{2}} \gamma_{+}^{p_{2}-p_{1}} x_{1}}{v^{p_{1}}}-Q^{-p_{2}} \gamma_{+}^{p_{2}}\right) \\
& =Q^{-p_{2}} \gamma_{+}^{p_{2}}\left(1-\frac{p_{2}}{p_{1}}\right)\left(\frac{x_{1}}{\left(\gamma_{+} v\right)^{p_{1}}}-1\right) .
\end{aligned}
$$

Observe that $\gamma_{+} v=a_{+}$and from Remark 2.3 we know that $\operatorname{sign}\left(x_{1}-a_{+}^{p_{1}}\right)=$ $-\operatorname{sign}\left(p_{1}\right)$. Therefore, the equation

$$
p_{1} \Pi=\frac{p_{1}}{v}\left(\frac{A x_{1}}{v^{p_{1}}}-\frac{x_{2}}{v^{p_{2}}}\right)=\frac{1}{v} Q^{-p_{2}} \gamma_{+}^{p_{2}}\left(p_{1}-p_{2}\right)\left(\frac{x_{1}}{a_{+}^{p_{1}}}-1\right)
$$

finishes the proof. 
Remark 2.12. One can draw a picture, take a point $x$, move it a little bit to the right and observe that $v^{p_{1}}$ has decreased. It exactly means that $v$ acts as claimed in the lemma. We give a picture for the case $p_{1}>p_{2}>0$. We encourage the reader to draw similar pictures for the other cases.

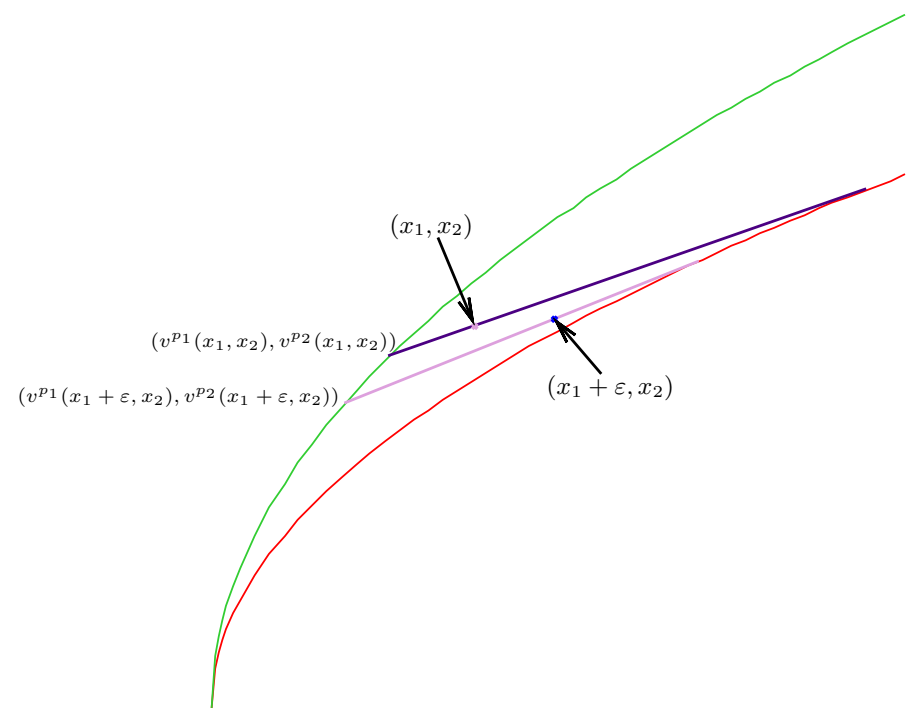

Figure 6: The illustration for $\operatorname{sign}\left(v_{x_{1}}^{\prime}\right)$.

Case 2: $x \in \Omega_{\mathrm{III}}$. We would like to do the same thing as before - study the sign of $v_{x_{1}}^{\prime}$. Note that it is obvious from the picture that $v_{x_{1}}^{\prime}$ behaves in the same way as before, but we shall prove it analytically.

Lemma 2.13. Let $v$ be as above and let $x \in \Omega_{\mathrm{III}}$. Then $\operatorname{sign} v^{\prime}{ }_{x_{1}}=-\operatorname{sign} p_{1}$ and $\operatorname{sign}\left(v_{x_{2}}^{\prime}\right)=\operatorname{sign}\left(p_{2}\right)$.

Proof. We have

$$
x_{1}-1=\frac{v^{p_{1}}-1}{v^{p_{2}}-1}\left(x_{2}-1\right) .
$$

Therefore, $1=\left(x_{2}-1\right) h^{\prime}(v) v_{x_{1}}^{\prime}$, where

$$
h(v)=\frac{v^{p_{1}}-1}{v^{p_{2}}-1} .
$$

Differentiating, we get

$$
\begin{aligned}
h^{\prime}(v) & =\frac{p_{1} v^{p_{1}-1}\left(v^{p_{2}}-1\right)-p_{2} v^{p_{2}-1}\left(v^{p_{1}}-1\right)}{\left(v^{p_{2}}-1\right)^{2}} \\
& =\frac{v^{p_{1}+p_{2}-1}}{\left(v^{p_{2}}-1\right)^{2}}\left(p_{1}-p_{2}+p_{2} v^{-p_{1}}-p_{1} v^{-p_{2}}\right)=\frac{v^{p_{1}+p_{2}-1}}{\left(v^{p_{2}}-1\right)^{2}} h_{1}(v),
\end{aligned}
$$

where

$$
h_{1}(v)=p_{1}-p_{2}+p_{2} v^{-p_{1}}-p_{1} v^{-p_{2}} .
$$


Thus,

$$
\operatorname{sign}\left(v_{x_{1}}^{\prime}\right)=\operatorname{sign}\left(x_{2}-1\right) \operatorname{sign}\left(h_{1}(v)\right) .
$$

Clearly, $\operatorname{sign}\left(x_{2}-1\right)=-\operatorname{sign}\left(p_{2}\right)$, and we only need to find $\operatorname{sign}\left(h_{1}(v)\right)$. Since

$$
h_{1}^{\prime}(v)=-p_{2} p_{1} v^{-p_{1}-1}+p_{1} p_{2} v^{-p_{2}-1}=p_{1} p_{2} v^{-p_{2}-1}\left(1-v^{p_{2}-p_{1}}\right),
$$

we have $\operatorname{sign}\left(h_{1}^{\prime}\right)=-\operatorname{sign}\left(p_{1} p_{2}\right)$. Note that $h_{1}(1)=0$, whence $\operatorname{sign}\left(h_{1}(v)\right)=$ $\operatorname{sign}\left(p_{1} p_{2}\right)$. Therefore, $\operatorname{sign}\left(v_{x_{1}}^{\prime}\right)=-\operatorname{sign}\left(p_{1}\right)$.

The result for $\operatorname{sign}\left(v_{x_{2}}^{\prime}\right)$ can be obtained similarly.

\subsection{On the local concavity and derivatives in the sense of distributions.}

In this part we are going to discuss the following question. Assume $B$ is not smooth, but still locally concave. How to express this in the sense of derivatives? The answer is easy: we must demand $\frac{d^{2} B}{d x^{2}} \leqslant 0$ in the sense of distributions. More precisely, the following theorem is true:

Theorem 2.14. The function $B$ is locally concave in $\Omega$ if and only if for every smooth function $\varphi \geqslant 0$ with a compact support in the interior of $\Omega$, and for every $\Delta_{1}, \Delta_{2} \in \mathbb{R}$ the following inequality holds:

$$
\int B(x)\left[\varphi_{x_{1} x_{1}}^{\prime \prime} \Delta_{1}^{2}+2 \varphi_{x_{1} x_{2}}^{\prime \prime} \Delta_{1} \Delta_{2}+\varphi_{x_{2} x_{2}}^{\prime \prime} \Delta_{2}^{2}\right] d x \leqslant 0 .
$$

Our next step is the following: we take one of the integrals above, for example $\int B(x) \varphi_{x_{1} x_{1}}^{\prime \prime}(x) d x$, and perform an integration by parts. While doing this, we assume $B$ is continuous. Although $B_{x_{1}}^{\prime}$ may not be, $B_{x_{1}}^{\prime}$ is piecewise differentiable.

Our motivation is the following: we are going to find a Bellman candidate $B$ that is twice differentiable in the interiors of $\Omega_{\mathrm{I}}, \Omega_{\mathrm{II}}, \Omega_{\mathrm{III}}$ and $\Omega_{\mathrm{IV}}$ and continuous at every point of $\ell_{ \pm}$. However, the first derivatives of $B$ may not be continuous on $\ell_{ \pm}$, and we want to catch the influence of their jumps on the integral above.

We state the following lemma, where $F$ plays the role of a derivative of $B$ :

Lemma 2.15. Let

$$
F\left(x_{1}, x_{2}\right)= \begin{cases}f_{1}\left(x_{1}, x_{2}\right), & x_{2} \geqslant k x_{1}+m, \\ f_{2}\left(x_{1}, x_{2}\right), & x_{2}<k x_{1}+m .\end{cases}
$$

Let $\varphi$ be a smooth function with compact support. By $(f, \varphi)$ we denote the action of the functional $f$ on the function $\varphi$. Then, considering $F_{x_{1}}$ and $F_{x_{2}}$ as distributions, we get

$$
\begin{aligned}
\left(F_{x_{1}}, \varphi\right)=\int & \int F_{x_{1}} \varphi d x_{1} d x_{2} \\
& \quad+k \int_{\mathbb{R}}\left(f_{2}\left(x_{1}, k x_{1}+m\right)-f_{1}\left(x_{1}, k x_{1}+m\right)\right) \varphi\left(x_{1}, k x_{1}+m\right) d x_{1}, \\
\left(F_{x_{2}}, \varphi\right)=\iint F_{x_{2}} \varphi d x_{1} d x_{2} & \\
& +\int_{\mathbb{R}}\left(f_{1}\left(x_{1}, k x_{1}+m\right)-f_{2}\left(x_{1}, k x_{1}+m\right)\right) \varphi\left(x_{1}, k x_{1}+m\right) d x_{1} .
\end{aligned}
$$


The proof of this lemma is simply integration by parts and we omit it. The following is an immediate corollary of Lemma 2.15 .

Remark 2.16. Take $F$ as above and assume $f_{1}\left(x_{1}, k x_{1}+m\right)$ is a constant $f_{1}$ and $f_{2}\left(x_{1}, k x_{1}+m\right)$ is a constant $f_{2}$. Then

$$
\begin{aligned}
\left(F_{x_{1}}, \varphi\right) & =\int F_{x_{1}} \varphi d x+k\left(f_{2}-f_{1}\right) \int \varphi\left(x_{1}, k x_{1}+m\right) d x_{1} ; \\
\left(F_{x_{2}}, \varphi\right) & =\int F_{x_{2}} \varphi d x+\left(f_{1}-f_{2}\right) \int \varphi\left(x_{1}, k x_{1}+m\right) d x_{1} .
\end{aligned}
$$

Finally, define $\Phi_{ \pm}(\varphi)=\int \varphi\left(x_{1}, Q^{-p_{2}} \frac{p_{2}}{p_{1}} \gamma_{ \pm}^{p_{2}-p_{1}}\left(x_{1}-1\right)+1\right) d x_{1}$. We simply integrate $\varphi$ over $\ell_{ \pm}$. Note that if $\varphi \geqslant 0$ then $\Phi_{ \pm}(\varphi) \geqslant 0$.

The second derivatives of $B$ will consist of two parts: the "classical" derivative and the functionals $\Phi_{ \pm}$. The second part corresponds to the "jump" of first derivatives.

To simplify our calculations we shall state a technical lemma. It says that to check that the "jump" matrix is nonpositive it is sufficient to check that the derivative is nonpositive only for one direction that is not parallel to $\ell_{ \pm}$.

Lemma 2.17. Let $B$ be as above, i.e. $\operatorname{det} \frac{d^{2} B}{d x^{2}}=0, B$ is a $C^{2}$ function in the interiors of $\Omega_{\mathrm{I}}-\Omega_{\mathrm{IV}}$, and the first derivatives of $B$ are constant on each $\ell_{ \pm}$. To check that the Hessian of $B$ is a nonpositive distribution it is sufficient to check that in interiors of $\Omega_{\mathrm{I}}-\Omega_{\mathrm{IV}}$ the Hessian is nonpositive and that $B_{x_{2} x_{2}}^{\prime \prime} \leqslant 0$ as a distribution.

We omit the proof of this lemma. The reader can find details in [7].

\subsection{On the approximation of $A_{p_{1}, p_{2}}^{Q}$-weights with bounded weights}

In this subsection we are going to prove two results about the approximation of $A_{p_{1}, p_{2}}^{Q}$-weights. The motivation is the following: if we have an integral of a function over a finite interval, it may be convenient to approximate the function by its bounded cut-offs, because to bounded functions we can apply the Lebesgue dominated convergence theorem.

We have the following lemma:

Lemma 2.18. Assume $w \in A_{p_{1}, p_{2}}^{Q}$. Take

$$
\underline{w}_{a}(t)= \begin{cases}w(t), & w(t) \leqslant a, \\ a, & w(t)>a .\end{cases}
$$

Then $\underline{w}_{a} \in A_{p_{1}, p_{2}}^{Q}$. The same is true for the function

$$
\bar{w}_{a}(t)= \begin{cases}a, & w(t) \leqslant a, \\ w(t), & w(t)>a .\end{cases}
$$


Remark 2.19. Note that

$$
\underline{w}_{a} \leqslant w \leqslant \bar{w}_{a}
$$

Remark 2.20. Note that it is sufficient to prove the lemma only for $\underline{w}_{a}$. The result for $\bar{w}_{a}$ will follow immediately, since instead of $w, p_{1}$, and $p_{2}$ we can consider $w^{-1}$, $-p_{2}$, and $-p_{1}$.

Proof. First, we fix an interval $J \subset[0,1]$ and write $J_{1}=\{t \in J: w(t) \leqslant a\}$ and $J_{2}=\{t \in J: w(t)>a\}$. Define also

$$
z_{i}=\left\langle w^{p_{1}}\right\rangle_{J_{i}}, \quad y_{i}=\left\langle w^{p_{2}}\right\rangle_{J_{i}}, \quad \alpha_{i}=\frac{\left|J_{i}\right|}{|J|} .
$$

Then we want to prove

$$
\begin{aligned}
& \left\langle w^{p_{1}}\right\rangle_{J}^{1 / p_{1}}\left\langle w^{p_{2}}\right\rangle_{J}^{-1 / p_{2}}-\left\langle w_{a}^{p_{1}}\right\rangle_{J}^{1 / p_{1}}\left\langle w_{a}^{p_{2}}\right\rangle_{J}^{-1 / p_{2}} \\
& =\left(\alpha_{1} z_{1}+\alpha_{2} z_{2}\right)^{1 / p_{1}}\left(\alpha_{1} y_{1}+\alpha_{2} y_{2}\right)^{-1 / p_{2}}-\left(\alpha_{1} z_{1}+\alpha_{2} a^{p_{1}}\right)^{1 / p_{1}}\left(\alpha_{1} y_{1}+\alpha_{2} a^{p_{2}}\right)^{-1 / p_{2}} \\
& \geqslant 0 .
\end{aligned}
$$

By Hölder's inequality, we get $z_{i}^{1 / p_{1}} \geqslant y_{i}^{1 / p_{2}}$. Therefore, if we denote $y_{2}^{1 / p_{2}}$ by $u$, then $z_{2}^{1 / p_{1}}=s u$ for a number $s \geqslant 1$ and the left-hand side of (2.4), which we need to estimate, can be written as the following function of $s$ and $u$ :

$$
\begin{aligned}
\varphi(s, u)= & \left(\alpha_{1} z_{1}+\alpha_{2} s^{p_{1}} u^{p_{1}}\right)^{1 / p_{1}}\left(\alpha_{1} y_{1}+\alpha_{2} u^{p_{2}}\right)^{-1 / p_{2}} \\
& -\left(\alpha_{1} z_{1}+\alpha_{2} a^{p_{1}}\right)^{1 / p_{1}}\left(\alpha_{1} y_{1}+\alpha_{2} a^{p_{2}}\right)^{-1 / p_{2}} .
\end{aligned}
$$

Since

$$
\frac{\partial \varphi}{\partial s}=\alpha_{2} s^{p_{1}-1} u^{p_{1}}\left(\alpha_{1} z_{1}+\alpha_{2} s^{p_{1}} u^{p_{1}}\right)^{1 / p_{1}-1} \geqslant 0
$$

the function $\varphi$ is increasing in $s$ and therefore $\varphi(s, u) \geqslant \varphi(1, u)$, i.e., it has its minimal value when $w(t)$ is equal to $u$ on $J_{2}$ identically.

Now we have $u=\left.w(t)\right|_{J_{2}}>a$ and since $\varphi(1, a)=0$, the desired inequality will be proved after checking that $\frac{\partial \varphi}{\partial u}(1, u) \geqslant 0$. We write

$$
\begin{aligned}
\frac{\partial \varphi}{\partial u}(1, u)= & \alpha_{2} u^{-1}\left(\alpha_{1} z_{1}+\alpha_{2} u^{p_{1}}\right)^{1 / p_{1}-1}\left(\alpha_{1} y_{1}+\alpha_{2} u^{p_{2}}\right)^{-1 / p_{2}-1} \\
& \cdot\left[u^{p_{1}}\left(\alpha_{1} y_{1}+\alpha_{2} u^{p_{2}}\right)-u^{p_{2}}\left(\alpha_{1} z_{1}+\alpha_{2} u^{p_{1}}\right)\right] \\
= & \alpha_{1} \alpha_{2} u^{-1}\left(\alpha_{1} z_{1}+\alpha_{2} u^{p_{1}}\right)^{1 / p_{1}-1}\left(\alpha_{1} y_{1}+\alpha_{2} u^{p_{2}}\right)^{-1 / p_{2}-1}\left[u^{p_{1}} y_{1}-u^{p_{2}} z_{1}\right],
\end{aligned}
$$

and this is what we need because $u^{p_{1}} y_{1}-u^{p_{2}} z_{1} \geqslant 0$. Indeed, since $u \geqslant w(t)$ and $p_{1} \geqslant p_{2}$, we have $u^{p_{1}-p_{2}} \geqslant w(t)^{p_{1}-p_{2}}$, whence $u^{p_{1}} w^{p_{2}} \geqslant u^{p_{2}} w^{p_{1}}$. Therefore,

$$
u^{p_{1}} y_{1}-u^{p_{2}} z_{1}=\left\langle u^{p_{1}} w^{p_{2}}-u^{p_{2}} w^{p_{1}}\right\rangle_{J_{1}} \geqslant 0
$$

which completes the proof. 


\section{Searching for $B$}

\subsection{Domain $\Omega_{\mathrm{I}}$}

This case was briefly discussed in Section 2.2.1.

Lemma 3.1. For every point $x=\left(x_{1}, x_{2}\right) \in \Omega_{\mathrm{I}}$ there are two numbers $u \geqslant 1$ and $v \geqslant 1$ such that $x$ lies on the line segment that connects $\left(u^{p_{1}}, u^{p_{2}}\right)$ and $\left(v^{p_{1}}, v^{p_{2}}\right)$, and this line segment lies in $\Omega_{\mathrm{I}}$.

This lemma is a simple geometrical observation. We refer the reader to Figures $1-3$.

Lemma 3.2. For every $x \in \Omega_{\mathrm{I}}$, we have $\mathcal{B}(x)=1$.

Proof of Lemma 3.2. Take a point $x \in \Omega_{\mathrm{I}}$ and the numbers $u$ and $v$ from Lemma 3.1. Then for some $\mu \in[0,1]$ we have $x_{k}=\mu u^{p_{k}}+(1-\mu) v^{p_{k}}$. Let

$$
w(t)= \begin{cases}u, & t \in[0, \mu), \\ v, & t \in[\mu, 1] .\end{cases}
$$

By Lemma 2.7, $w \in A_{p_{1}, p_{2}}^{Q}$. Further,

$$
\left\langle w^{p_{k}}\right\rangle=\mu u^{p_{k}}+(1-\mu) v^{p_{k}}=x_{k} .
$$

We have $u, v \geqslant 1$, thus $|\{w(t) \geqslant 1\}|=1$. Since $\left\langle w^{p_{k}}\right\rangle=x_{k}$, and $w \in A_{p_{1}, p_{2}}^{Q}$, we get

$$
\mathcal{B}(x) \geqslant|\{w(t) \geqslant 1\}|=1 \text {. }
$$

On the other hand, by definition, $\mathcal{B}(x) \leqslant 1$. Therefore, $\mathcal{B}(x)=1$.

\subsection{Domain $\Omega_{\mathrm{III}}$}

In this section we find $B$ in $\Omega_{\mathrm{III}}$. We discussed our plan in Section 2.2.1, and now we are going to give the full details. As was said in Section 1.6, we need to find lines on which $B$ is linear. These lines will simply be the lines connecting the point $(1,1)$ with points on $\Gamma$. Our setting is the following: we fix a point $\left(v^{p_{1}}, v^{p_{2}}\right) \in \Gamma$ and consider the line with the equation

$$
v^{p_{2}}\left(1-x_{1}\right)-v^{p_{1}}\left(1-x_{2}\right)=x_{2}-x_{1} .
$$

Clearly, this line contains the points $(1,1)$ and $\left(v^{p_{1}}, v^{p_{2}}\right)$. We assume that $B$ is linear on our line. Using that $B(1,1)=1$ and $B\left(v^{p_{1}}, v^{p_{2}}\right)=0$, we get

$$
B(x)=\frac{x_{1}-v^{p_{1}}}{1-v^{p_{1}}}=\frac{x_{2}-v^{p_{2}}}{1-v^{p_{2}}} .
$$




\subsection{Domain $\Omega_{\mathrm{II}}$}

To find $B$ in $\Omega_{\mathrm{II}}$, we use the following simple observation. The boundary of $\Omega_{\mathrm{II}}$ has three parts: the parts of $\ell_{ \pm}$and a part of $\Gamma_{Q}$. Since our candidate for $\mathcal{B}$ is linear on the mentioned parts of $\ell_{ \pm}$, it is natural to assume $B$ to be linear in the whole of $\Omega_{\mathrm{II}}$, namely, $B(x)=a x_{1}+b x_{2}+c$.

We would like to explain the word "natural". In the paper [7] this is called an optimality principle of building Monge-Ampère foliations. The rough idea behind it is that the smallest concave function is linear; and since we have two linear boundaries of $\Omega_{\mathrm{II}}$, on which $B$ is linear, we have a hope that we can extend $B$ to a linear function in the whole $\Omega_{\mathrm{II}}$.

We now show how to find $a, b$ and $c$. We want $B$ to be continuous on $\ell_{ \pm}$and, therefore, we want $B(1,1)=1, B\left(v_{-}^{p_{1}}, v_{-}^{p_{2}}\right)=0$ and $B\left(v_{+}^{p_{1}}, v_{+}^{p_{2}}\right)=1$. This gives us three equations:

$$
\left\{\begin{array}{l}
a+b+c=1, \\
a v_{-}^{p_{1}}+b v_{-}^{p_{2}}+c=0, \\
a v_{+}^{p_{1}}+b v_{+}^{p_{2}}+c=1 .
\end{array}\right.
$$

Solving this linear system (and using $v_{+}=1 / v_{-}$) one gets

$$
\left\{\begin{array}{l}
a=\frac{v_{-}^{p_{1}}}{\left(1-v_{-}^{p_{1}}\right)\left(v_{-}^{p_{1}}-v_{-}^{p_{2}}\right)}, \\
b=\frac{v_{-}^{p_{2}}}{\left(v_{-}^{p_{2}}-1\right)\left(v_{-}^{p_{1}}-v_{-}^{p_{2}}\right)}, \\
c=1-\frac{1}{\left(v_{-}^{p_{1}}-1\right)\left(v_{-}^{p_{2}}-1\right)} .
\end{array}\right.
$$

\subsection{Domain $\Omega_{\mathrm{IV}}$}

Now we shall find $B$ in $\Omega_{\mathrm{IV}}$. We guess that if $x \in \Omega_{\mathrm{IV}}$ then $B$ is linear on the tangent from $x$ to $\Gamma_{Q}$, which corresponds to $\gamma_{+}$.

Let us give an explanation. Past experience building Bellman functions, see [6], [7], [9], and [8], shows that in a lot of cases the Bellman foliation consists exactly of these tangent lines (this is called tangency principle in [7]). Since we do not have any other guesses, we should check this one.

We remind the reader what our guess means. For every point $x$ there is a unique point $\left(v^{p_{1}}, v^{p_{2}}\right) \in \Gamma_{1}$, such that $x$ lies on the line through this point that is tangent to $\Gamma_{Q}$ at $\left(\left(\gamma_{+} v\right)^{p_{1}},\left(\frac{\gamma_{+} v}{Q}\right)^{p_{2}}\right)$. Namely, the equation of this tangent is

$$
x_{2}=Q^{-p_{2}} \frac{p_{2}}{p_{1}} \gamma_{+}^{p_{2}-p_{1}} v^{p_{2}-p_{1}}\left(x_{1}-v^{p_{1}}\right)+v^{p_{2}} .
$$

We know that on this line $t_{0}, t_{1}$, and $t_{2}$ are supposed to be constants. This means that they depend only on $v$. Therefore, we divide the equation

$$
d t_{0}+x_{1} d t_{1}+x_{2} d t_{2}=0
$$


by $d v$ and get:

$$
t_{0}^{\prime}(v)+x_{1} t_{1}^{\prime}(v)+x_{2} t_{2}^{\prime}(v)=0
$$

when

$$
x_{2}=Q^{-p_{2}} \frac{p_{2}}{p_{1}} \gamma_{+}^{p_{2}-p_{1}} v^{p_{2}-p_{1}}\left(x_{1}-v^{p_{1}}\right)+v^{p_{2}} .
$$

Now we substitute $x_{2}$ from this equation into (3.1) and use that for a fixed $v$ equation (3.1) is true for all $x_{1}$ between $v^{p_{1}}$ and $\left(\gamma_{+} v\right)^{p_{1}}$. Therefore, the coefficient of $x_{1}$ must be equal to zero, which yields

$$
t_{1}^{\prime}+Q^{-p_{2}} \frac{p_{2}}{p_{1}} \gamma_{+}^{p_{2}-p_{1}} v^{p_{2}-p_{1}} t_{2}^{\prime}=0
$$

Since $B\left(v^{p_{1}}, v^{p_{2}}\right)=0$, we get

$$
t_{0}+v^{p_{1}} t_{1}+v^{p_{2}} t_{2}=0, d t_{0}+v^{p_{1}} d t_{1}+v^{p_{2}} d t_{2}=0,
$$

SO

$$
d t_{0}+v^{p_{1}} d t_{1}+p_{1} v^{p_{1}-1} t_{1} d v+v^{p_{2}} d t_{2}+p_{2} v^{p_{2}-1} t_{2} d v=0
$$

or

$$
p_{1} t_{1} v^{p_{1}-1}+p_{2} t_{2} v^{p_{2}-1}=0
$$

thus

$$
t_{2}^{\prime}=-\frac{p_{1}}{p_{2}} v^{p_{1}-p_{2}-1}\left(t_{1}^{\prime} v+\left(p_{1}-p_{2}\right) t_{1}\right) .
$$

Combining the last equation with (3.2) we obtain

$$
t_{1}^{\prime}\left(1-Q^{-p_{2}} \gamma_{+}^{p_{2}-p_{1}}\right)=\frac{p_{1}-p_{2}}{v} t_{1} Q^{-p_{2}} \gamma_{+}^{p_{2}-p_{1}},
$$

So

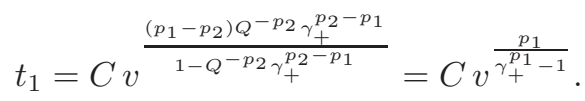

From (3.3) we get

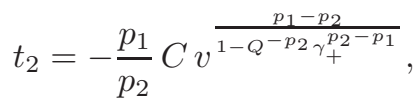

and from $t_{0}+v^{p_{1}} t_{1}+v^{p_{2}} t_{2}=0$ we get

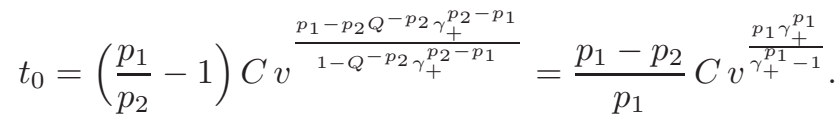

We shall find $C$ such that $B$ is continuous in $\Omega_{\mathrm{III}} \cap \Omega_{\mathrm{IV}}$. As before, $A=Q^{-p_{2}} \gamma_{+}^{p_{2}-p_{1}}$. On the line

$$
x_{2}=A \frac{p_{2}}{p_{1}} v_{-}^{p_{2}-p_{1}} x_{1}+v_{-}^{p_{2}}\left(1-\frac{p_{2}}{p_{1}} A\right)
$$


we have

$$
\begin{aligned}
& B\left(x_{1}, x_{2}\right)=C\left[\frac{p_{1}-p_{2}}{p_{2}} v_{-}^{\frac{p_{1}-p_{2} A}{1-A}}+x_{1} v_{-}^{\frac{\left(p_{1}-p_{2}\right) A}{1-A}}-A \frac{p_{2}}{p_{1}} v_{-}^{p_{2}-p_{1}} x_{1} \frac{p_{1}}{p_{2}} v_{-}^{\frac{p_{1}-p_{2}}{1-A}}\right.\left.-\frac{p_{1}-p_{2} A}{p_{1}} v_{-}^{p_{2}} \frac{p_{1}}{p_{2}} v_{-}^{\frac{p_{1}-p_{2}}{1-A}}\right] \\
&=C(1-A)\left[x_{1} v_{-}^{\frac{\left(p_{1}-p_{2}\right) A}{1-A}}-v_{-}^{\frac{p_{1}-p_{2} A}{1-A}}\right] .
\end{aligned}
$$

However, on this line

So

$$
B\left(x_{1}, x_{2}\right)=\frac{x_{1}-v_{-}^{p_{1}}}{1-v_{-}^{p_{1}}}
$$

$$
C=\frac{1}{1-A} \frac{v_{-}^{-\frac{\left(p_{1}-p_{2}\right) A}{1-A}}}{1-v_{-}^{p_{1}}}
$$

\subsection{The formula for $B$}

Now we state the unified formula for $B$.

$$
B(x)= \begin{cases}1, & x \in \Omega_{\mathrm{I}}, \\ \frac{v_{-}^{p_{1}}}{\left(1-v_{-}^{p_{1}}\right)\left(v_{-}^{p_{1}}-v_{-}^{p_{2}}\right)} x_{1}+\frac{v_{-}^{p_{2}}}{\left(v_{-}^{p_{2}}-1\right)\left(v_{-}^{p_{1}}-v_{-}^{p_{2}}\right)} x_{2}+1-\frac{1}{\left(v_{-}^{p_{1}}-1\right)\left(v_{-}^{p_{2}}-1\right)}, & x \in \Omega_{\mathrm{II}}, \\ \frac{x_{1}-v^{p_{1}}}{1-v^{p_{1}}}, & x \in \Omega_{\mathrm{III}}, \\ \frac{1}{1-A} \frac{v_{-}^{-\frac{\left(p_{1}-p_{2}\right) A}{1-A}}}{1-v_{-}^{p_{1}}} v^{\frac{p_{1}-p_{2}}{1-A}}\left(\frac{p_{1}-p_{2}}{p_{2}} v^{p_{2}}+x_{1} v^{p_{2}-p_{1}}-\frac{p_{1}}{p_{2}} x_{2}\right), & x \in \Omega_{\mathrm{IV}},\end{cases}
$$

where $v$ is defined as the solution of equation

$$
\begin{cases}v^{p_{2}}\left(1-x_{1}\right)-v^{p_{1}}\left(1-x_{2}\right)=x_{2}-x_{1}, & x \in \Omega_{\mathrm{III}}, \\ x_{2}=Q^{-p_{2}} \frac{p_{2}}{p_{1}} \gamma_{+}^{p_{2}-p_{1}} v^{p_{2}-p_{1}}\left(x_{1}-v^{p_{1}}\right)+v^{p_{2}}, & x \in \Omega_{\mathrm{IV}},\end{cases}
$$

that has the property that $\operatorname{sign}\left(x_{1}-v^{p_{1}}\right)=\operatorname{sign}\left(p_{1}\right)$.

\section{The estimate from above: $B \geqslant \mathcal{B}$}

To state the main theorem of this section we need to introduce some notation. Recall that in the definition of $B$ there was a fixed number $Q>1$. In fact, we could write

$$
\mathcal{B}_{Q}\left(x_{1}, x_{2}\right)=\sup \left\{|\{t: w(t) \geqslant 1\}|:\left\langle w^{p_{1}}\right\rangle=x_{1},\left\langle w^{p_{2}}\right\rangle=x_{2}, w \in A_{p_{1}, p_{2}}^{Q}\right\},
$$

and to denote our Bellman candidate $B_{Q}$. We had dropped the index $Q$ for simplicity, but now we need it. 
We now take a number $Q_{1}>Q$ and take the corresponding Bellman candidate $B_{Q_{1}}$, as defined in (3.4) (but for $Q_{1}$ instead of $Q$ ). The function $B_{Q_{1}}$ is defined on the domain $\Omega_{Q_{1}}$, which contains $\Omega_{Q}$.

The main result of this section is the following theorem:

Theorem 4.1. For every point $x \in \Omega_{Q}$ and for every $Q_{1}>Q$ we have that $B_{Q_{1}}(x) \geqslant \mathcal{B}_{Q}(x)$.

It is easy to check that $B_{Q_{1}}$ is continuous in $Q_{1}$, which immediately gives the following corollary:

Corollary 4.2. For every point $x \in \Omega_{Q}$ we have that $B(x) \geqslant \mathcal{B}(x)$.

To prove the Theorem 4.1 we need the following two lemmata. The lengthy proof of the first one is postponed until Section 5. The proof of the second one can be found in [9].

Lemma 4.3. The function $B_{Q}$ is locally concave.

Lemma 4.4. Fix $Q_{1}>Q>1$. Then for every $w \in A_{p_{1}, p_{2}}^{Q}$ there are two intervals $I^{+}$and $I^{-}$such that $I=I^{-} \cup I^{+}$and if $x^{ \pm}=\left(\left\langle w^{p_{1}}\right\rangle_{I_{ \pm}},\left\langle w^{p_{2}}\right\rangle_{I_{ \pm}}\right)$then $\left[x^{-}, x^{+}\right] \subset \Omega_{Q_{1}}$. Also the parameters $\alpha^{ \pm}$can be taken separated from 0 and 1 uniformly with respect to $w$.

Proof of Theorem 4.1. We want to prove that for any function $w \in A_{p_{1}, p_{2}}^{Q}(I)$ and $x=\left(\left\langle w^{p_{1}}\right\rangle,\left\langle w^{p_{2}}\right\rangle\right)$ it is true that

$$
B_{Q_{1}}(x) \geqslant|\{w \geqslant 1\}| \text {. }
$$

Then, taking the supremum of the right-hand side, we get what we need. Assume $w \in A_{p_{1}, p_{2}}^{Q}$. We take a splitting of our interval $I$ by the rule from Lemma 4.4 ; then we split $I_{ \pm}$according to the same rule and continue the splitting process. By $D_{n}$ we denote the set of intervals of the $n$-th generation. Thus $D_{0}=\{I\}, D_{1}=\left\{I^{-}, I^{+}\right\}$, and so on. For every interval $J \in D_{n}$ we let

$$
x^{J}=\left(\left\langle w^{p_{1}}\right\rangle_{J},\left\langle w^{p_{2}}\right\rangle_{J}\right) .
$$

Since $B_{Q_{1}}$ is locally concave, we get

$$
\begin{aligned}
B_{Q_{1}}(x) & \geqslant\left|I^{-}\right| B_{Q_{1}}\left(x^{I^{-}}\right)+\left|I^{+}\right| B_{Q_{1}}\left(x^{I^{+}}\right) \\
& \geqslant \sum_{J \in D_{n}}|J| B_{Q_{1}}\left(x^{J}\right)=\int_{0}^{1} B_{Q_{1}}\left(x^{n}(t)\right) d t,
\end{aligned}
$$

where $x^{n}(t)$ is a step function defined in the following way: for each $J \in D_{n}$ let $x^{n}(t)=x^{J}, t \in J$.

Since we assume that $w^{p_{i}} \in L_{1, l o c}$, we get

$$
x^{n}(t) \rightarrow\left(w^{p_{1}}(t), w^{p_{2}}(t)\right) \quad \text { a.e. }
$$


Moreover, in Section 6 it will be proved that for every $x \in \Omega$ there exists a function $w \in A_{p_{1}, p_{2}}^{Q}$ such that $B(x)=|\{w \geqslant 1\}|$. The same can be shown for $Q_{1}$ instead of $Q$, so we get $B_{Q_{1}}(x) \leqslant 1$. Therefore, by the Lebesgue dominated convergence theorem, we can pass to the limit in (4.2). Then we get

$$
B_{Q_{1}}(x) \geqslant \int_{0}^{1} B_{Q_{1}}\left(w^{p_{1}}(t), w^{p_{2}}(t)\right) d t .
$$

However, for every $t$ we have $\left(w^{p_{1}}(t), w^{p_{2}}(t)\right) \in \Gamma_{1}$, where we know $B_{Q_{1}}$ by Lemma 1.3. Therefore,

$$
B_{Q_{1}}(x) \geqslant|\{t: w(t) \geqslant 1\}|
$$

which is what we need.

\section{Proof of concavity}

This section is devoted to the proof of Lemma 4.3. We recall its statement.

Lemma. The following inequality holds in the sense of distributions:

$$
\frac{d^{2} B}{d x^{2}} \leqslant 0
$$

We break the proof of this lemma into parts. Following Section 2.4, first we check that in interiors of $\Omega_{\mathrm{I}}-\Omega_{\mathrm{IV}}$ the Hessian of $B$ is nonpositive.

Then we study the jumps of $B_{x_{2}}^{\prime}$ across the boundaries between the subdomains.

We warn the reader that this section is rather technical.

\subsection{Domains $\Omega_{\mathrm{I}}$ and $\Omega_{\mathrm{II}}$}

Here $B$ is fully linear and, therefore, $\frac{d^{2} B}{d x^{2}}=0$.

\subsection{Domain $\Omega_{\mathrm{III}}$}

As we know, here

$$
B(x)=\frac{x_{2}-v^{p_{2}}}{1-v^{p_{2}}}=\frac{x_{2}-1}{1-v^{p_{2}}}+1
$$

Recall that $v^{p_{2}}\left(1-x_{1}\right)-v^{p_{1}}\left(1-x_{2}\right)=x_{2}-x_{1}$, so

$$
\left(p_{2} v^{p_{2}-1}\left(1-x_{1}\right)-p_{1} v^{p_{1}-1}\left(1-x_{2}\right)\right) v_{x_{1}}^{\prime}-v^{p_{2}}=-1,
$$

or

$$
v_{x_{1}}^{\prime}=v \frac{v^{p_{2}}-1}{\Upsilon}
$$

where

$$
\Upsilon=\Upsilon(v)=p_{2} v^{p_{2}}\left(1-x_{1}\right)-p_{1} v^{p_{1}}\left(1-x_{2}\right) .
$$


Put

$$
f(v)=\frac{v^{p_{2}}}{v^{p_{2}}-1}=1+\frac{1}{v^{p_{2}}-1} .
$$

Differentiating (5.1), we get

$$
\begin{aligned}
B_{x_{1}}^{\prime} & =\left(x_{2}-1\right) \cdot \frac{p_{2} v^{p_{2}-1}}{\left(1-v^{p_{2}}\right)^{2}} v_{x_{1}}^{\prime}=p_{2}\left(x_{2}-1\right) \cdot \frac{v^{p_{2}-1}}{\left(1-v^{p_{2}}\right)^{2}} \cdot \frac{v\left(v^{p_{2}}-1\right)}{\Upsilon} \\
& =p_{2}\left(x_{2}-1\right) \cdot \frac{v^{p_{2}}}{v^{p_{2}}-1} \cdot \frac{1}{\Upsilon}=p_{2}\left(x_{2}-1\right) \frac{f(v)}{\Upsilon} .
\end{aligned}
$$

Observe that

$$
f_{x_{1}}^{\prime}(v)=-p_{2} \cdot \frac{v^{p_{2}-1}}{\left(v^{p_{2}}-1\right)^{2}} \cdot v_{x_{1}}^{\prime}=-p_{2} \cdot \frac{f(v)}{\Upsilon}
$$

Therefore,

$$
\begin{aligned}
& B_{x_{1} x_{1}}^{\prime \prime}=p_{2}\left(x_{2}-1\right)\left[-p_{2} \frac{f(v)}{\Upsilon^{2}}-\frac{\left(p_{2}^{2} v^{p_{2}-1}\left(1-x_{1}\right)-p_{1}^{2} v^{p_{1}-1}\left(1-x_{2}\right)\right) v_{x_{1}}^{\prime}-p_{2} v^{p_{2}}}{\Upsilon^{2}} f(v)\right] \\
& =\frac{p_{2}\left(1-x_{2}\right) f(v)}{\Upsilon^{2}}\left[p_{2}-p_{2} v^{p_{2}}+\left(p_{2}^{2} v^{p_{2}-1}\left(1-x_{1}\right)-p_{1}^{2} v^{p_{1}-1}\left(1-x_{2}\right)\right) \frac{v\left(v^{p_{2}}-1\right)}{\Upsilon}\right] \\
& =-\frac{p_{2}\left(x_{2}-1\right) f(v)}{\Upsilon^{2}}\left(v^{p_{2}}-1\right)\left[\frac{p_{2}^{2} v^{p_{2}}\left(1-x_{1}\right)-p_{1}^{2} v^{p_{1}}\left(1-x_{2}\right)}{p_{2} v^{p_{2}}\left(1-x_{1}\right)-p_{1} v^{p_{1}}\left(1-x_{2}\right)}-p_{2}\right] \\
& =-\frac{p_{2}\left(x_{2}-1\right) f(v)\left(v^{p_{2}}-1\right)}{\Upsilon^{2}} \cdot \frac{p_{2} p_{1} v^{p_{1}}\left(1-x_{2}\right)-p_{1}^{2} v^{p_{1}}\left(1-x_{2}\right)}{\Upsilon} \\
& =-\frac{p_{1} p_{2}\left(x_{2}-1\right) f(v)\left(v^{p_{2}}-1\right) v^{p_{1}}\left(1-x_{2}\right)\left(p_{2}-p_{1}\right)}{\Upsilon^{3}} \\
& =\frac{\left(x_{2}-1\right)^{2}\left(p_{2}-p_{1}\right) v^{p_{1}+p_{2}}}{\Upsilon^{2}} \cdot \frac{p_{1} p_{2}}{\Upsilon} .
\end{aligned}
$$

Now we calculate $B_{x_{2} x_{2}}^{\prime \prime}$. We use that the expression (5.1) can be rewritten as

$$
B(x)=\frac{x_{1}-v^{p_{1}}}{1-v^{p_{1}}}=\frac{x_{1}-1}{1-v^{p_{1}}}+1 .
$$

By a straightforward calculation we get

$$
B_{x_{2} x_{2}}^{\prime \prime}=\frac{\left(x_{1}-1\right)^{2}\left(p_{2}-p_{1}\right) v^{p_{1}+p_{2}}}{\Upsilon^{2}} \frac{p_{1} p_{2}}{\Upsilon}
$$

Using that $\operatorname{det} \frac{d^{2} B}{d x^{2}}=0$, we immediately get

$$
B_{x_{1} x_{2}}^{\prime \prime}= \pm \frac{\left(1-x_{1}\right)\left(1-x_{2}\right) v^{p_{1}+p_{2}}\left(p_{2}-p_{1}\right)}{\Upsilon^{2}} \frac{p_{1} p_{2}}{\Upsilon}
$$

and the sign is not important.

Finally,

$$
\sum_{i, j} B_{x_{i} x_{j}}^{\prime \prime} \Delta_{i} \Delta_{j}=\frac{v^{p_{1}+p_{2}}\left(p_{2}-p_{1}\right)}{\Upsilon^{2}} \frac{p_{1} p_{2}}{\Upsilon}\left(\left(1-x_{1}\right) \Delta_{1} \pm\left(1-x_{2}\right) \Delta_{2}\right)^{2}
$$


Recall that

$$
\Upsilon=v \frac{v^{p_{2}}-1}{v_{x_{1}}^{\prime}}
$$

so

$$
\begin{gathered}
\sum_{i, j} B_{x_{i} x_{j}}^{\prime \prime} \Delta_{i} \Delta_{j}=\frac{v^{p_{1}+p_{2}}\left(p_{2}-p_{1}\right)}{\Upsilon^{2}} \frac{1}{v} \frac{p_{1} p_{2} v_{x_{1}}^{\prime}}{v^{p_{2}}-1}\left(\left(1-x_{1}\right) \Delta_{1} \pm\left(1-x_{2}\right) \Delta_{2}\right)^{2} \\
=\frac{v^{p_{1}+p_{2}}\left(p_{2}-p_{1}\right)}{\Upsilon^{2}} \frac{1}{v} \frac{p_{2}}{v^{p_{2}}-1} p_{1} v_{x_{1}}^{\prime}\left(\left(1-x_{1}\right) \Delta_{1} \pm\left(1-x_{2}\right) \Delta_{2}\right)^{2}
\end{gathered}
$$

Observe that $\operatorname{sign}\left(v^{p_{2}}-1\right)=-\operatorname{sign}\left(p_{2}\right), \operatorname{sign} v_{x_{1}}^{\prime}=-\operatorname{sign} p_{1}$ and $p_{2}-p_{1}<0$. This gives

$$
\sum_{i, j} B_{x_{i} x_{j}}^{\prime \prime} \Delta_{i} \Delta_{j} \leqslant 0
$$

\subsection{Domain $\Omega_{\mathrm{IV}}$}

From Section 3.4 we know that

$$
B_{x_{1}}^{\prime}=t_{1}=\frac{1}{1-A} \frac{v_{-}^{-\alpha}}{1-v_{-}^{p_{1}}} v^{\frac{\left(p_{1}-p_{2}\right) A}{1-A}} .
$$

(We do not need to write the full expression for $\alpha$.) Moreover, put $V_{-}=\frac{v_{-}^{-\alpha}}{1-v_{-}^{p_{1}}}$. Then we get

$$
B_{x_{1} x_{1}}^{\prime \prime}=\frac{\left(p_{1}-p_{2}\right) A}{(1-A)^{2}} V_{-} v^{\frac{\left(p_{1}-p_{2}\right) A}{1-A}-1} v_{x_{1}}^{\prime}
$$

Similarly,

$$
\begin{aligned}
B_{x_{2}}^{\prime} & =t_{2}=-\frac{p_{1}}{p_{2}} \frac{1}{1-A} V_{-} v^{\frac{p_{1}-p_{2}}{1-A}}, \\
B_{x_{2} x_{2}}^{\prime \prime} & =-\frac{p_{1}}{p_{2}} \frac{p_{1}-p_{2}}{(1-A)^{2}} V_{-} v^{\frac{p_{1}-p_{2}}{1-A}-1} v_{x_{2}}^{\prime}, \\
B_{x_{1} x_{2}}^{\prime \prime} & =B_{x_{2} x_{1}}^{\prime \prime}=-\frac{p_{1}}{p_{2}} \frac{p_{1}-p_{2}}{(1-A)^{2}} V_{-} v^{\frac{p_{1}-p_{2}}{1-A}-1} v_{x_{1}}^{\prime} .
\end{aligned}
$$

As we know from Lemma 2.11,

$$
v_{x_{1}}^{\prime}=\frac{1}{p_{1} \Pi} \frac{A}{v^{p_{1}}}, \quad v_{x_{2}}^{\prime}=-\frac{1}{p_{2} \Pi} \frac{1}{v^{p_{2}}} .
$$

We have

$$
\operatorname{sign}\left(B_{x_{1} x_{1}}^{\prime \prime}\right)=\operatorname{sign}\left(1-v_{-}^{p_{1}}\right) \operatorname{sign}\left(v_{x_{1}}^{\prime}\right)=-1 .
$$

Similarly $\operatorname{sign}\left(B_{x_{2} x_{2}}^{\prime \prime}\right)=-1$, and since $\operatorname{det}\left(\frac{d^{2} B}{d x^{2}}\right)=0$, we get that $\frac{d^{2} B}{d x^{2}} \leqslant 0$. 


\subsection{Boundary}

Now we proceed to the "jumps" of the first derivatives of $B$ across the splitting lines $\ell_{ \pm}$. We recall that in $\Omega_{\mathrm{II}}$ we have

$$
B(x)=a x_{1}+b x_{2}+c,
$$

where

$$
b=\frac{v_{-}^{p_{2}}}{\left(v_{-}^{p_{2}}-1\right)\left(v_{-}^{p_{1}}-v_{-}^{p_{2}}\right)} .
$$

We also recall the functionals $\Phi_{ \pm}$from Section 2.4 that act on smooth compactly supported functions $\varphi$ as follows:

$$
\Phi_{ \pm}(\varphi)=\int \varphi\left(x_{1}, Q^{-p_{2}} \frac{p_{2}}{p_{1}} \gamma_{ \pm}^{p_{2}-p_{1}}\left(x_{1}-1\right)+1\right) d x_{1}
$$

5.4.1. Boundary $\boldsymbol{\Omega}_{\mathbf{I}} \cap \boldsymbol{\Omega}_{\mathbf{I I}}$. Observe that if $p_{2}>0$ then

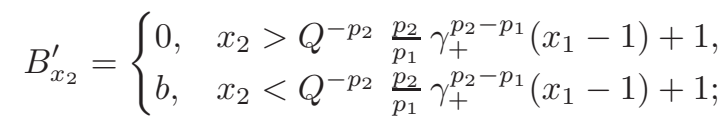

and if $p_{2}<0$ then

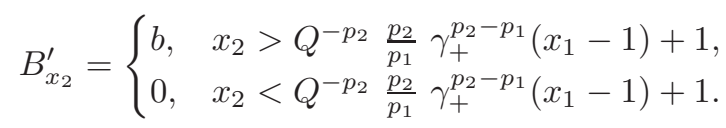

Therefore, in the sense of distributions,

$$
B_{x_{2} x_{2}}^{\prime \prime}=-\operatorname{sign}\left(p_{2}\right) b \Phi_{+} .
$$

Notice that $\Phi_{+}$is a nonnegative functional, and therefore the sign of $B_{x_{2} x_{2}}^{\prime \prime}$ is determined by the $\operatorname{sign}$ of $-\operatorname{sign}\left(p_{2}\right) b$. Since

$$
b=\frac{v_{-}^{p_{2}}}{\left(v_{-}^{p_{2}}-1\right)\left(v_{-}^{p_{1}}-v_{-}^{p_{2}}\right)},
$$

and

$$
\begin{aligned}
\operatorname{sign}\left(v_{-}^{p_{2}}-1\right) & =-\operatorname{sign}\left(p_{2}\right), \\
v_{-}^{p_{1}}-v_{-}^{p_{2}} & =v_{-}^{p_{1}}\left(1-v_{-}^{p_{2}-p_{1}}\right)<0,
\end{aligned}
$$

we get $\operatorname{sign}(b)=\operatorname{sign}\left(p_{2}\right)$, so

$$
B_{x_{2} x_{2}}^{\prime \prime} \leqslant 0
$$

5.4.2. Boundary $\Omega_{\mathrm{II}} \cap \Omega_{\mathrm{III}}$. Our plan is the following. First we calculate $B_{x_{2}}^{\prime}$ on the line $\frac{x_{2}-1}{v_{-}^{p 2}-1}=\frac{x_{1}-1}{v_{-}^{p 1}-1}$, i.e., on $\ell_{-}$. Then we proceed to the jumps in the derivatives. 

we get

Using (5.1) and the inequalities $\operatorname{sign}\left(1-v_{-}^{p_{1}}\right)=\operatorname{sign}\left(p_{1}\right)$ and $1-Q^{-p_{2}} \gamma_{+}^{p_{2}-p_{1}}>0$,

$$
B_{x_{2}}^{\prime}\left(\ell_{-}\right)=-\frac{p_{1}}{p_{2}} \cdot \frac{1}{1-Q^{-p_{2}} \gamma_{+}^{p_{2}-p_{1}}} \frac{v_{-}^{p_{1}-p_{2}}}{1-v_{-}^{p_{1}}},
$$

and

$$
\operatorname{sign}\left(B_{x_{2}}^{\prime}\left(\ell_{-}\right)\right)=-\operatorname{sign} p_{2} .
$$

As before, we observe that if $p_{2}>0$ then

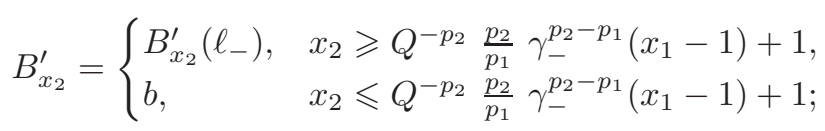

and if $p_{2}<0$ then

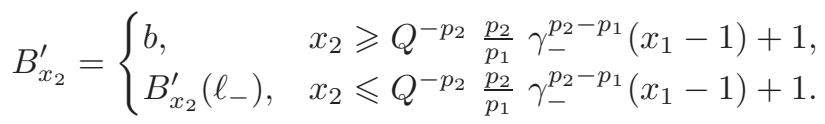

Therefore,

$$
\left(B_{x_{2} x_{2}}^{\prime \prime}, \varphi\right)=\operatorname{sign}\left(p_{2}\right)\left(B_{x_{2}}^{\prime}\left(\ell_{-}\right)-b\right) \Phi_{-}(\varphi)+\int B_{x_{2} x_{2}}^{\prime \prime} \varphi d x
$$

Moreover,

$$
\operatorname{sign}\left(B_{x_{2}}^{\prime}\left(\ell_{-}\right)\right)=-\operatorname{sign}\left(p_{2}\right) \quad \text { and } \quad \operatorname{sign}(b)=\operatorname{sign}\left(p_{2}\right),
$$

So

$$
\operatorname{sign}\left(p_{2}\right)\left(B_{x_{2}}^{\prime}\left(\ell_{-}\right)-b\right) \leqslant 0 .
$$

This completes the consideration of the boundary $\Omega_{\mathrm{II}} \cap \Omega_{\mathrm{III}}$.

5.4.3. Boundary $\boldsymbol{\Omega}_{\mathbf{I I I}} \cap \boldsymbol{\Omega}_{\mathbf{I V}}$. This is the best boundary since here all the derivatives of $B$ are continuous. We check this by a straightforward calculation. We remark that due to Lemma 2.17, it is sufficient to check the continuity of only one derivative, namely, $B_{x_{2}}^{\prime}$.

We already know the values of $B_{x_{2}}^{\prime}$ when we approach $\ell_{-}$from $\Omega_{\mathrm{III}}$. Observe that in $\Omega_{\mathrm{IV}}$

$$
B(x)=\frac{1}{1-A} \frac{v_{-}^{-\frac{\left(p_{1}-p_{2}\right) A}{1-A}}}{1-v_{-}^{p_{1}}} v^{\frac{p_{1}-p_{2}}{1-A}}\left(\frac{p_{1}-p_{2}}{p_{2}} v^{p_{2}}+x_{1} v^{p_{2}-p_{1}}-\frac{p_{1}}{p_{2}} x_{2}\right) .
$$

Also we know that the solution of the Monge-Ampère equation satisfies the following: $B_{x_{2}}^{\prime}=t_{2}$. Thus, using that $A=Q^{-p_{2}} \gamma_{-}^{p_{2}-p_{1}}$, we get

$$
t_{2}=-\frac{p_{1}}{p_{2}} \frac{1}{1-A} \frac{v_{-}^{-\frac{\left(p_{1}-p_{2}\right) A}{1-A}}}{1-v_{-}^{p_{1}}} v^{\frac{p_{1}-p_{2}}{1-A}}
$$

so

$$
t_{2}\left(v_{-}\right)=-\frac{p_{1}}{p_{2}} \frac{1}{1-A} \frac{v_{-}^{p_{1}-p_{2}}}{1-v_{-}^{p_{1}}}=B_{x_{2}}\left(\ell_{-}\right),
$$

which finishes the consideration of the boundary $\Omega_{\mathrm{III}} \cap \Omega_{\mathrm{IV}}$. 


\section{The estimate from below: $B \leqslant \mathcal{B}$. Constructing opti- mizers}

In this section we construct an optimizer for each point $x \in \Omega$. Namely, for each point $x \in \Omega$ we find a function $w=w_{x}$ with the following properties:

1) $\left\langle w^{p_{1}}\right\rangle=x_{1},\left\langle w^{p_{2}}\right\rangle=x_{2}$;

2) $w \in A_{p_{1}, p_{2}}^{Q}$;

3) $|\{t: w(t) \geqslant 1\}|=B(x)$.

The existence of the optimizer means that $\mathcal{B}(x) \geqslant\left|\left\{t: w_{x}(t) \geqslant 1\right\}\right|=B(x)$, which, coupled with the estimate $\mathcal{B}(x) \geqslant B(x)$, will complete the process of finding the Bellman function $\mathcal{B}$.

As usual, we break our proof into four parts, each pertaining to a specific subdomain of $\Omega$.

\subsection{Domain $\Omega_{\mathrm{I}}$}

In Subsection 3.1 we have already proved that for every point $x \in \Omega_{\mathrm{I}}$ there is a suitable function $w$ such that $|\{t: w(t) \geqslant 1\}|=1$.

\subsection{Domain $\Omega_{\mathrm{II}}$}

We proceed with the idea from Subsection 2.2.1. Take $x \in \Omega_{\text {II }}$ and a segment $\left[x^{-}, x^{+}\right] \subset \Omega_{\text {II }}$ such that $x \in\left[x^{-}, x^{+}\right]$and $x^{ \pm} \in \ell_{ \pm}$. We write

$$
x=\lambda x^{+}+(1-\lambda) x^{-}
$$

for some $\lambda \in[0,1]$. We also can write that

$$
x_{i}^{ \pm}=\mu_{ \pm}+\left(1-\mu_{ \pm}\right) v_{ \pm}^{p_{i}}
$$

for some $\mu_{ \pm} \in[0,1]$. We know that we can choose the following function as optimizers for $x^{ \pm}$:

$$
w^{-}(t)=\left\{\begin{array}{ll}
v_{-}, & t \in\left[0,1-\mu_{-}\right), \\
1, & t \in\left[1-\mu_{-}, 1\right] ;
\end{array} \quad w^{+}(t)= \begin{cases}1, & t \in\left[0, \mu_{+}\right) \\
v_{+}, & t \in\left[\mu_{+}, 1\right]\end{cases}\right.
$$

The functions $w^{ \pm}$are defined in this mirror-like fashion (the constant 1 is assigned to the right part of $[0,1]$ for $w^{-}$and to the left part of $[0,1]$ for $\left.w^{+}\right)$, because we are about to glue them in the proportion dictated by (6.1). Also, we want to glue them in a way that will minimize the $A_{p_{1}, p_{2}}$-characteristic of the resulting compound test function for $x$. More specifically:

$$
w(t)= \begin{cases}v_{-}, & t \in\left[0,(1-\lambda)\left(1-\mu_{-}\right)\right] \\ 1, & t \in\left((1-\lambda)\left(1-\mu_{-}\right), 1-\lambda+\lambda \mu_{+}\right], \\ v_{+}, & t \in\left(1-\lambda+\lambda \mu_{+}, 1\right] .\end{cases}
$$


Note that

$$
\begin{aligned}
\left\langle w^{p_{i}}\right\rangle= & v_{-}^{p_{i}}(1-\lambda)\left(1-\mu_{-}\right)+1-\lambda+\lambda \mu_{+}-(1-\lambda) \\
& +(1-\lambda) \mu_{-}+v_{+}^{p_{i}}\left(\lambda-\lambda \mu_{+}\right) \\
= & (1-\lambda)\left(\mu_{-}+\left(1-\mu_{-}\right) v_{-}^{p_{i}}\right)+\lambda\left(\mu_{+}+\left(1-\mu_{+}\right) v_{+}^{p_{i}}\right)=x_{i} .
\end{aligned}
$$

Now our goal is to prove that for every $[\alpha, \beta] \subset[0,1]$ we have

$$
\left\langle w^{p_{1}}\right\rangle_{[\alpha, \beta]}^{1 / p_{1}}\left\langle w^{p_{2}}\right\rangle_{[\alpha, \beta]}^{-1 / p_{2}} \leqslant Q,
$$

and that $B(x)=|\{t: w(t) \geqslant 1\}|$. This follows from the next two lemmas and the fact that we have chosen $x^{ \pm}$so that the segment $\left[x^{-}, x^{+}\right]$lies inside the domain $\Omega$.

Lemma 6.1. The point $\left(\left\langle w^{p_{1}}\right\rangle_{[\alpha, \beta]},\left\langle w^{p_{2}}\right\rangle_{[\alpha, \beta]}\right)$ is a convex combination of $x^{-}, x^{+}$ and $(1,1)$ or lies on $\ell_{ \pm}$.

Lemma 6.2. $B(x)=|\{t: w(t) \geqslant 1\}|$.

Proof of Lemma 6.1. The proof of this lemma is similar to the proof of Lemma 2.7.

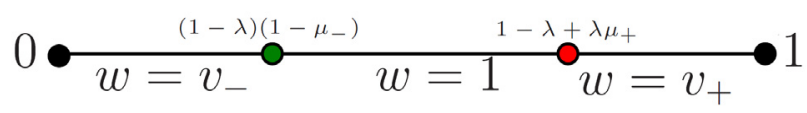

It is easy to see that the only interesting case is $\alpha<(1-\lambda)\left(1-\mu_{-}\right)$and $\beta>1-\lambda+\lambda \mu_{+}$. In the other cases we have a convex combination of $v_{-}$and $(1,1)$ or a convex combination of $v_{+}$and $(1,1)$, which were treated in Lemma 2.7. If $\alpha<(1-\lambda)\left(1-\mu_{-}\right)$and $\beta>1-\lambda+\lambda \mu_{+}$then

$$
\begin{aligned}
\left\langle w^{p_{i}}\right\rangle_{[\alpha, \beta]}= & \frac{1}{\beta-\alpha}\left[v_{-}^{p_{i}}\left((1-\lambda)\left(1-\mu_{-}\right)-\alpha\right)+\left(\lambda \mu_{+}+(1-\lambda) \mu_{-}\right)\right. \\
& \left.+v_{+}^{p_{i}}\left(\beta-1+\lambda-\lambda \mu_{+}\right)\right] \\
= & \frac{1}{\beta-\alpha}\left[\frac{\left((1-\lambda)\left(1-\mu_{-}\right)-\alpha\right)}{1-\mu_{-}} \cdot x_{i}^{-}+\frac{\beta-1+\lambda-\lambda \mu_{+}}{1-\mu_{+}} \cdot x_{i}^{+}\right. \\
& \left.+\left(\alpha \frac{\mu_{-}}{1-\mu_{-}}+(1-\beta) \frac{\mu_{+}}{1-\mu_{+}}\right) \cdot 1\right] .
\end{aligned}
$$

Note that the sum of all coefficients is equal to one, so we have a convex combination of $x^{ \pm}$and $(1,1)$.

Proof of Lemma 6.2. Since $B$ is linear in $\operatorname{clos}\left(\Omega_{\mathrm{II}}\right)$ we get

$$
\begin{aligned}
B(x)= & \lambda B\left(x^{+}\right)+(1-\lambda) B\left(x^{-}\right) \\
= & \lambda\left(1-\mu_{+}\right) B\left(v_{+}^{p_{1}}, v_{+}^{p_{2}}\right)+\lambda \mu_{+} B(1,1)+(1-\lambda) \mu_{-} B(1,1) \\
& +(1-\lambda)\left(1-\mu_{-}\right) B\left(v_{-}^{p_{1}}, v_{-}^{p_{2}}\right) \\
= & \lambda\left(1-\mu_{+}\right)+\lambda \mu_{+}+(1-\lambda) \mu_{-}=\lambda+(1-\lambda) \mu_{-} .
\end{aligned}
$$


On the other hand,

$$
|\{w \geqslant 1\}|=1-(1-\lambda)\left(1-\mu_{-}\right)=\lambda+\mu_{-}-\lambda \mu_{-},
$$

which finishes the proof.

\subsection{Domain $\Omega_{I I I}$}

Take a point $x \in \Omega_{\text {III }}$ and connect it with the point $(1,1)$. Take a number $v<1$, such that the point $\left(v^{p_{1}}, v^{p_{2}}\right)$ lies on the line segment that connects $(1,1)$ and $x$. Then there is a number $\mu \in[0,1]$ such that $x_{1}=\mu+(1-\mu) v^{p_{1}}$, and $x_{2}=$ $\mu+(1-\mu) v^{p_{2}}$. Define

$$
w(t)= \begin{cases}1, & t \in[0, \mu), \\ v, & t \in[\mu, 1] .\end{cases}
$$

By Lemma 2.7, $w \in A_{p_{1}, p_{2}}^{Q}$; moreover, it is easy to see that $\left\langle w^{p_{k}}\right\rangle=x_{k}$ (this follows from the definition of $\mu$ ).

Moreover, since $v<1$, we get

$$
\mathcal{B}(x) \geqslant|\{t: w(t) \geqslant 1\}|=\mu=\frac{x_{1}-v^{p_{1}}}{1-v^{p_{1}}}=B(x) .
$$

\subsection{Domain $\Omega_{\mathrm{IV}}$}

Our plan is the following: first we consider $x \in \Gamma_{Q}$; after we build a suitable function $w$ for every such $x$, it will be easy to construct an optimizer for every $x \in \Omega_{\mathrm{IV}}$.

6.4.1. The case $\boldsymbol{x} \in \boldsymbol{\Gamma}_{\boldsymbol{Q}}$. Take $x \in \Gamma_{Q} \cap \Omega_{\mathrm{IV}}$. We have

$$
x_{1}=\gamma_{+}^{p_{1}} v^{p_{1}} \quad \text { and } \quad x_{2}=Q^{-p_{2}} \gamma_{+}^{p_{2}} v^{p_{2}}
$$

for some $v<1$. To introduce $w$ we need some notation. First, choose $\nu$ such that

$$
\frac{1}{1-\nu p_{1}}=\gamma_{+}^{p_{1}}
$$

Take now

$$
a=\left(\frac{v}{v_{-}}\right)^{1 / \nu}
$$

We also recall that

$$
v_{-}=\frac{\gamma_{-}}{\gamma_{+}}
$$

Now define

$$
w(t)= \begin{cases}1, & t \in\left[0, \frac{\gamma_{-}^{p_{1}}-v_{-}^{p_{1}}}{1-v_{-}^{p_{1}}} \cdot a\right), \\ v_{-}, & t \in\left[\frac{\gamma_{-}^{p_{1}}-v_{-}^{p_{-}}}{1-v_{-}^{p_{1}}} \cdot a, a\right), \\ v_{-}\left(\frac{a}{t}\right)^{\nu}, & t \in[a, 1] .\end{cases}
$$

We check that $w$ has the desired properties. 
Lemma 6.3. $\left\langle w^{p_{k}}\right\rangle=x_{k}$.

Lemma 6.4. $w \in A_{p_{1}, p_{2}}^{Q}$.

Lemma 6.5. $|\{w \geqslant 1\}|=B(x)$.

We prove Lemma 6.4 in Section 7. Let us prove Lemmas 6.3 and 6.5.

Proof of Lemma 6.3. For $k=1$ we make a direct calculation:

$$
\begin{aligned}
\left\langle w^{p_{1}}\right\rangle & =\frac{\gamma_{-}^{p_{1}}-v_{-}^{p_{1}}}{1-v_{-}^{p_{1}}} a+v_{-}^{p_{1}} \frac{1-\gamma_{-}^{p_{1}}}{1-v_{-}^{p_{1}}} a+v_{-}^{p_{1}} a^{\nu p_{1}} \frac{1}{1-\nu p_{1}}\left(1-a^{1-\nu p_{1}}\right) \\
& =\gamma_{-}^{p_{1}} a+v_{-}^{p_{1}} a^{\nu p_{1}} \frac{1}{1-\nu p_{1}}-v_{-}^{p_{1}} a \frac{1}{1-\nu p_{1}} \\
& =\gamma_{-}^{p_{1}} a+v^{p_{1}} \gamma_{+}^{p_{1}}-v_{-}^{p_{1}} \gamma_{+}^{p_{1}} a=\gamma_{-}^{p_{1}} a+x_{1}-\gamma_{-}^{p_{1}} a=x_{1} .
\end{aligned}
$$

For $k=2$ we need the following:

$$
\frac{1}{1-\nu p_{2}}=Q^{-p_{2}} \gamma_{+}^{p_{2}}
$$

To prove it take equation (2.1):

$$
Q^{-p_{2}}\left(1-\frac{p_{2}}{p_{1}}\right) \gamma_{+}^{p_{2}}=1-\frac{p_{2}}{p_{1}} Q^{-p_{2}} \gamma_{+}^{p_{2}-p_{1}} .
$$

Multiplying this by $Q^{p_{2}} \gamma_{+}^{-p_{2}}$ we get:

$$
1-\frac{p_{2}}{p_{1}}=Q^{p_{2}} \gamma_{+}^{-p_{2}}-\frac{p_{2}}{p_{1}} \gamma_{+}^{-p_{1}}=Q^{p_{2}} \gamma_{+}^{-p_{2}}-\frac{p_{2}}{p_{1}}\left(1-\nu p_{1}\right),
$$

so

$$
Q^{p_{2}} \gamma_{+}^{-p_{2}}=1-\frac{p_{2}}{p_{1}}+\frac{p_{2}}{p_{1}}-\nu p_{2}=1-\nu p_{2},
$$

which is what we need.

Observe also that the points $(1,1),\left(\gamma_{-}^{p_{1}}, Q^{-p_{2}} \gamma_{-}^{p_{2}}\right)$ and $\left(v_{-}^{p_{1}}, v_{-}^{p_{2}}\right)$ lie on the line $\ell_{-}$. Therefore, we have the following equation:

$$
\frac{\gamma_{-}^{p_{1}}-v_{-}^{p_{1}}}{1-v_{-}^{p_{1}}}=\frac{Q^{-p_{2}} \gamma_{-}^{p_{2}}-v_{-}^{p_{2}}}{1-v_{-}^{p_{2}}} .
$$

The calculation for $\left\langle w^{p_{2}}\right\rangle$ is almost identical to the one for $\left\langle w^{p_{1}}\right\rangle$, and we omit it.

Equation (6.5) is very useful for us, so we want to put it as a separate lemma.

Lemma 6.6. In our notation, we have

$$
\frac{1}{1-\nu p_{1}}=\gamma_{+}^{p_{1}} \quad \text { and } \quad \frac{1}{1-\nu p_{2}}=Q^{-p_{2}} \gamma_{+}^{p_{2}} .
$$

Consequently,

$$
x_{k}=\frac{v^{p_{k}}}{1-\nu p_{k}} .
$$


Proof of Lemma 6.5. Since $w$ is a decreasing function and $v_{-}<1$, we get

$$
|\{w \geqslant 1\}|=\frac{\gamma_{-}^{p_{1}}-v_{-}^{p_{1}}}{1-v_{-}^{p_{1}}} \cdot a .
$$

Let us compute $B(x)$. This will be a direct calculation. Specifically, substituting $x_{k}=\frac{v^{p_{k}}}{1-\nu p_{k}}$ into the fourth line of (3.4), we get

$$
\begin{aligned}
B(x) & =\frac{1}{1-A} \frac{v_{-}^{-\frac{\left(p_{1}-p_{2}\right) A}{1-A}}}{1-v_{-}^{p_{1}}} v^{\frac{p_{1}-p_{2}}{1-A}}\left(\frac{p_{1}-p_{2}}{p_{2}} v^{p_{2}}+\frac{1}{1-\nu p_{1}} v^{p_{2}}-\frac{p_{1}}{p_{2}} \frac{1}{1-\nu p_{2}} v^{p_{2}}\right) \\
& =\frac{1}{1-A} \frac{v_{-}^{-\frac{\left(p_{1}-p_{2}\right) A}{1-A}}}{1-v_{-}^{p_{1}}} v^{\frac{p_{1}-p_{2} A}{1-A}}\left(\frac{p_{1}}{p_{2}}-1+\frac{1}{1-\nu p_{1}}-\frac{p_{1}}{p_{2}} \frac{1}{1-\nu p_{2}}\right) \\
& =\frac{1}{1-A} \frac{v_{-}^{-\frac{\left(p_{1}-p_{2}\right) A}{1-A}}}{1-v_{-}^{p_{1}}} v^{\frac{p_{1}-p_{2} A}{1-A}}\left(\frac{\nu p_{1}}{1-\nu p_{1}}-\frac{p_{1}}{p_{2}} \frac{\nu p_{2}}{1-\nu p_{2}}\right) \\
& =\frac{1}{1-A} \frac{v_{-}^{-\frac{\left(p_{1}-p_{2}\right) A}{1-A}}}{1-v_{-}^{p_{1}}} v^{\frac{p_{1}-p_{2} A}{1-A}}\left(\frac{\nu p_{1}}{1-\nu p_{1}}-\frac{\nu p_{1}}{1-\nu p_{2}}\right) \\
& =\frac{1}{1-A} \frac{v_{-}^{-\frac{\left(p_{1}-p_{2}\right) A}{1-A}}}{1-v_{-}^{p_{1}}} v^{\frac{p_{1}-p_{2} A}{1-A}} \nu p_{1} \frac{\nu p_{1}-\nu p_{2}}{\left(1-\nu p_{1}\right)\left(1-\nu p_{2}\right)} .
\end{aligned}
$$

Recall that $A=Q^{-p_{2}} \gamma_{+}^{p_{2}-p_{1}}$, so

$$
1-A=1-\gamma_{+}^{-p_{1}} Q^{-p_{2}} \gamma_{+}^{p_{2}}=1-\frac{1-\nu p_{1}}{1-\nu p_{2}}=\frac{\nu p_{1}-\nu p_{2}}{1-\nu p_{2}} .
$$

Therefore,

$$
B(x)=\frac{v_{-}^{-\frac{\left(p_{1}-p_{2}\right) A}{1-A}}}{1-v_{-}^{p_{1}}} v^{\frac{p_{1}-p_{2} A}{1-A}} \nu p_{1} \frac{1}{\left(1-\nu p_{1}\right)} .
$$

Moreover, observe that

$$
v_{-}^{-\frac{\left(p_{1}-p_{2}\right) A}{1-A}}=v_{-}^{-\frac{p_{1}-p_{2} A}{1-A}} v_{-}^{p_{1}}
$$

SO

$$
B(x)=\frac{a^{\nu \cdot \frac{p_{1}-p_{2} A}{1-A}}}{1-v_{-}^{p_{1}}} v_{-}^{p_{1}} \frac{\nu p_{1}}{1-\nu p_{1}}=\frac{a^{\nu \cdot \frac{p_{1}-p_{2} A}{1-A}}}{1-v_{-}^{p_{1}}} \frac{\gamma_{-}^{p_{1}}}{\gamma_{+}^{p_{1}}} \nu p_{1} \gamma_{+}^{p_{1}}=\frac{\nu p_{1} \gamma_{-}^{p_{1}}}{1-v_{-}^{p_{1}}} a^{\nu \frac{p_{1}-p_{2} A}{1-A}} .
$$

Furthermore,

$$
p_{1}-p_{2} A=p_{1}-p_{2} \frac{1-\nu p_{1}}{1-\nu p_{2}}=\frac{p_{1}-p_{2}}{1-\nu p_{2}},
$$

thus

$$
\frac{p_{1}-p_{2} A}{1-A}=\frac{1}{\nu}
$$


Using this we get

$$
B(x)=\frac{\nu p_{1} \cdot \gamma_{-}^{p_{1}}}{1-v_{-}^{p_{1}}} a=\frac{\gamma_{-}^{p_{1}}+\left(\nu p_{1}-1\right) \gamma_{-}^{p_{1}}}{1-v_{-}^{p_{1}}} a=\frac{\gamma_{-}^{p_{1}}-\gamma_{+}^{-p_{1}} \gamma_{-}^{p_{1}}}{1-v_{-}^{p_{1}}} a=\frac{\gamma_{-}^{p_{1}}-v_{-}^{p_{1}}}{1-v_{-}^{p_{1}}} a,
$$

and that is exactly what we need.

6.4.2. The case of arbitrary $\boldsymbol{x} \in \boldsymbol{\Omega}_{\mathrm{IV}}$. We now take an $x \in \Omega_{\mathrm{IV}}$ and the point $\left(v^{p_{1}}, v^{p_{2}}\right) \in \Gamma$ such that $x \in \ell_{+}(v)$. Let $y=\left(\gamma_{+}^{p_{1}} v^{p_{1}}, Q^{-p_{2}} \gamma_{+}^{p_{2}} v^{p_{2}}\right)$. Let $w_{y}$ be the function defined as in (6.4). Note that there is a number $\lambda \in[0,1]$ such that

$$
\begin{aligned}
& x_{1}=(1-\lambda) v^{p_{1}}+\lambda v^{p_{1}} \gamma_{+}^{p_{1}}, \\
& x_{2}=(1-\lambda) v^{p_{2}}+\lambda Q^{-p_{2}} v^{p_{2}} \gamma_{+}^{p_{2}} .
\end{aligned}
$$

Define now

$$
w(t)= \begin{cases}w_{y}\left(\frac{t}{\lambda}\right), & t \in[0, \lambda] \\ v, & t \in(\lambda, 1]\end{cases}
$$

Let $w_{y}^{\lambda}(t)=w_{y}\left(\frac{t}{\lambda}\right)$. This function is defined when $t \leqslant \lambda$, but when $t$ is close to $\lambda$, it is a power function, so we can extend it to the interval $[0,1]$, keeping it in $A_{p_{1}, p_{2}}^{Q}$. So we assume now that our $w_{y}^{\lambda}(t)$ is defined for $t \in[0,1]$. We note that

$$
w(t)= \begin{cases}w_{y}^{\lambda}(t), & w_{y}\left(\frac{t}{\lambda}\right) \geqslant v \\ v, & w_{y}\left(\frac{t}{\lambda}\right) \leqslant v\end{cases}
$$

Therefore, by Lemma 2.18, $w \in A_{p_{1}, p_{2}}^{Q}$.

Moreover, since $B$ is linear on $\ell_{+}(v)$ and since $v<1$, we get

$$
B(x)=(1-\lambda) B\left(v^{p_{1}}, v^{p_{2}}\right)+\lambda B(y)=\lambda B(y)=\lambda\left|\left\{w_{y}(t) \geqslant 1\right\}\right|=|\{w(t) \geqslant 1\}|,
$$

which finishes our proof.

\section{Calculating the $A_{p_{1}, p_{2}}$-characteristic of the test function}

In this section we prove that the $A_{p_{1}, p_{2}}$-characteristic of the weight $w$, defined in (6.4), is bounded by $Q$. For convenience we recall some notation from the previous section. We fix a point $x=\left(x_{1}, x_{2}\right)=\left(\gamma_{+}^{p_{1}} v^{p_{1}}, Q^{-p_{2}} \gamma_{+}^{p_{2}} v^{p_{2}}\right) \in \Gamma_{Q}$. First we take a number $\nu$ such that

$$
\frac{1}{1-\nu p_{1}}=\gamma_{+}^{p_{1}}
$$

We know that

$$
\frac{1}{1-\nu p_{2}}=Q^{-p_{2}} \gamma_{+}^{p_{2}}
$$


Consequently,

$$
x_{k}=\frac{v^{p_{k}}}{1-\nu p_{k}} \text {. }
$$

We recall that

$$
v_{-}=\frac{\gamma_{-}}{\gamma_{+}}
$$

and take

$$
a=\left(\frac{v}{v_{-}}\right)^{1 / \nu}
$$

Now we define

$$
w(t)= \begin{cases}1, & t \in\left[0, \frac{\gamma_{-}^{p_{1}}-v_{-}^{p_{1}}}{1-v_{-}^{p_{1}}} \cdot a\right), \\ v_{-}, & t \in\left[\frac{\gamma_{-}^{p_{1}}-v_{-}^{p_{-}}}{1-v_{-}^{p_{1}}} \cdot a, a\right), \\ v_{-} \cdot\left(\frac{a}{t}\right)^{\nu}, & t \in[a, 1] .\end{cases}
$$

Let

$$
u(t)=v_{-}\left(\frac{a}{t}\right)^{\nu}, \quad t \in[0,1] .
$$

Our first lemma is the following:

Lemma 7.1. $u \in A_{p_{1}, p_{2}}^{Q}$.

This lemma was proved in [9], but we repeat the proof.

Proof. We take an interval $J=[\alpha, \beta]$ and write

$$
\begin{aligned}
\left\langle u^{p_{1}}\right\rangle_{J}^{1 / p_{1}}\left\langle u^{p_{2}}\right\rangle_{J}^{-1 / p_{2}} & =\left(x_{1} \cdot \frac{\beta^{1-\nu p_{1}}-\alpha^{1-\nu p_{1}}}{\beta-\alpha}\right)^{1 / p_{1}}\left(x_{2} \cdot \frac{\beta^{1-\nu p_{2}}-\alpha^{1-\nu p_{2}}}{\beta-\alpha}\right)^{-1 / p_{2}} \\
& =Q\left(\frac{\beta^{1-\nu p_{1}}-\alpha^{1-\nu p_{1}}}{\beta-\alpha}\right)^{1 / p_{1}}\left(\frac{\beta^{1-\nu p_{2}}-\alpha^{1-\nu p_{2}}}{\beta-\alpha}\right)^{-1 / p_{2}} .
\end{aligned}
$$

To prove that the left-hand side is not greater than $Q$ we now have to prove the following estimate for every $\alpha$ and $\beta$, such that $0 \leqslant \alpha \leqslant \beta \leqslant 1$ :

$$
\left(\frac{\beta^{1-\nu p_{1}}-\alpha^{1-\nu p_{1}}}{\beta-\alpha}\right)^{1 / p_{1}}\left(\frac{\beta^{1-\nu p_{2}}-\alpha^{1-\nu p_{2}}}{\beta-\alpha}\right)^{-1 / p_{2}} \leqslant 1 .
$$

Let $s=\alpha / \beta$. Then the left-hand side of this inequality is equal to

$$
g(s, \nu):=\left(\frac{1-s^{1-\nu p_{1}}}{1-s}\right)^{1 / p_{1}}\left(\frac{1-s^{1-\nu p_{2}}}{1-s}\right)^{-1 / p_{2}},
$$

where $0 \leqslant s \leqslant 1$. Then

$$
\frac{\partial g}{\partial \nu}=\text { something positive } \cdot \log (s) \cdot\left(1-s^{\nu p_{1}-\nu p_{2}}\right) \leqslant 0,
$$

and therefore

$$
g(s, \nu) \leqslant g(s, 0)=1
$$


Let

$$
\underline{u}_{v_{-}}(t)= \begin{cases}v_{-}, & t \in[0, a], \\ v_{-} \cdot(a / t)^{\nu}, & t \in[a, 1] .\end{cases}
$$

From Lemma 2.18 we know that $\underline{u}_{v_{-}} \in A_{p_{1}, p_{2}}^{Q}$. Recall that to prove that the initial function $w$ is also in $A_{p_{1}, p_{2}}^{Q}$, we have to prove that for every interval $J \subset[0,1]$

$$
\left\langle w^{p_{1}}\right\rangle_{J}^{1 / p_{1}}\left\langle w^{p_{2}}\right\rangle_{J}^{-1 / p_{2}} \leqslant Q
$$

But from Lemmas 7.1 and 2.7 we already know this for many intervals $J$. The only intervals that remain to be investigated are those of the form $J=[\alpha, \beta]$ with $\alpha<\frac{\gamma_{-}^{p_{1}}-v_{-}^{p_{1}}}{1-v_{-}^{p_{1}}} \cdot a, \beta>a$. This will be our last step.

Lemma 7.2. If $J=[\alpha, \beta]$ and $\alpha<\frac{\gamma_{-}^{p_{1}}-v_{-}^{p_{1}}}{1-v_{-}^{p_{1}}} \cdot a, \beta>a$, then

$$
\left\langle w^{p_{1}}\right\rangle_{J}^{1 / p_{1}}\left\langle w^{p_{2}}\right\rangle_{J}^{-1 / p_{2}} \leqslant Q
$$

Proof. We have,

$$
\begin{aligned}
\left\langle w^{p_{1}}\right\rangle_{J}=\frac{1}{\beta-\alpha}\left[\left(\frac{\gamma_{-}^{p_{1}}-v_{-}^{p_{1}}}{1-v_{-}^{p_{1}}} \cdot a-\alpha\right)+v_{-}^{p_{1}}\right. & a \frac{1-\gamma_{-}^{p_{1}}}{1-v_{-}^{p_{1}}}+ \\
& \left.+\frac{v^{p_{1}}}{1-\nu p_{1}}\left(\beta^{1-\nu p_{1}}-a^{1-\nu p_{1}}\right)\right] .
\end{aligned}
$$

Note that

$$
x_{1}=\left\langle w^{p_{1}}\right\rangle=\frac{\gamma_{-}^{p_{1}}-v_{-}^{p_{1}}}{1-v_{-}^{p_{1}}} a+v_{-}^{p_{1}} a \frac{1-\gamma_{-}^{p_{1}}}{1-v_{-}^{p_{1}}}+\frac{v^{p_{1}}}{1-\nu p_{1}}\left(1-a^{1-\nu p_{1}}\right),
$$

and

$$
x_{1}=\frac{v^{p_{1}}}{1-\nu p_{1}}
$$

Therefore,

$$
\left\langle w^{p_{1}}\right\rangle_{J}=\frac{x_{1} \beta^{1-\nu p_{1}}-\alpha}{\beta-\alpha},
$$

and, similarly,

$$
\left\langle w^{p_{2}}\right\rangle_{J}=\frac{x_{2} \beta^{1-\nu p_{2}}-\alpha}{\beta-\alpha} .
$$

Therefore,

$$
\left\langle w^{p_{1}}\right\rangle_{J}^{1 / p_{1}}\left\langle w^{p_{2}}\right\rangle_{J}^{-1 / p_{2}}=\left(\frac{x_{1} \beta^{1-\nu p_{1}}-\alpha}{\beta-\alpha}\right)^{1 / p_{1}}\left(\frac{x_{2} \beta^{1-\nu p_{2}}-\alpha}{\beta-\alpha}\right)^{-1 / p_{2}} .
$$

Let

$$
F(\alpha, \beta)=\left(\frac{x_{1} \beta^{1-\nu p_{1}}-\alpha}{\beta-\alpha}\right)^{1 / p_{1}}\left(\frac{x_{2} \beta^{1-\nu p_{2}}-\alpha}{\beta-\alpha}\right)^{-1 / p_{2}}
$$


be the right-hand side of the last expression. First, we introduce new variables:

$$
t=\frac{x_{1}}{\beta^{\nu p_{1}}}, \quad \text { and } \quad s=\frac{\alpha}{\beta} .
$$

Define

$$
G(s, t)=F(\alpha, \beta)=\left(\frac{t-s}{1-s}\right)^{1 / p_{1}}\left(\frac{Q^{-p_{2}} t^{\frac{p_{2}}{p_{1}}}-s}{1-s}\right)^{-1 / p_{2}} .
$$

We prove the following lemma:

Lemma 7.3. $G$ does not attain its maximum in the interior of its domain.

Remark 7.4. We avoid writing the domain of $G$ explicitly. However, its domain has some obvious properties. For example, $0 \leqslant s<1$ and

$$
\left(\frac{t-s}{1-s}\right)=\frac{x_{1} \beta^{1-\nu p_{1}}-\alpha}{\beta-\alpha}=\left\langle w^{p_{1}}\right\rangle_{J}>0,
$$

thus $t>s$.

Proof of Lemma 7.3. G is a smooth function, so if it has a maximum in the interior of its domain, then at this point both $G_{t}^{\prime}$ and $G_{s}^{\prime}$ are equal to zero. Let

$$
M=\frac{t-s}{1-s} \quad \text { and } \quad N=\frac{Q^{-p_{2}} t^{\frac{p_{2}}{p_{1}}}-s}{1-s} .
$$

Then

$$
M_{t}^{\prime}=\frac{1}{1-s} \quad \text { and } \quad N_{t}^{\prime}=\frac{p_{2}}{p_{1}} Q^{-p_{2}} t^{\frac{p_{2}}{p_{1}}-1} \frac{1}{1-s} .
$$

Therefore, $G_{t}^{\prime}=0$ if and only if

$$
N-M Q^{-p_{2}} t^{\frac{p_{2}}{p_{1}}-1}=0
$$

which yields

$$
\left(Q^{-p_{2}} t^{\frac{p_{2}}{p_{1}}}-s\right)-(t-s) Q^{-p_{2}} t^{\frac{p_{2}}{p_{1}}-1}=0
$$

and, therefore,

$$
s\left(Q^{-p_{2}} t^{\frac{p_{2}}{p_{1}}-1}-1\right)=0 .
$$

Since in the interior of the domain $s>0$, we get $Q^{-p_{2}} t^{\frac{p_{2}}{p_{1}}-1}=1$. Note that then $t \neq 1$.

Now let us compute the partial derivative with respect to $s$, assuming that the last equality holds. We have

$$
M_{s}^{\prime}=\frac{t-1}{(s-1)^{2}} \quad \text { and } \quad N_{s}^{\prime}=\frac{Q^{-p_{2}} t^{\frac{p_{2}}{p_{1}}}-1}{(s-1)^{2}}=M_{s}^{\prime} .
$$

Since $t \neq 1$, we have

$$
G_{s}^{\prime}=\frac{1}{p_{1}} M^{1 / p_{1}-1} N^{-1 / p_{2}} M_{s}^{\prime}-\frac{1}{p_{2}} M^{1 / p_{1}} N^{-1 / p_{2}-1} N_{s}^{\prime} .
$$


If $G_{s}^{\prime}=0$, then

$$
\frac{N}{p_{1}}-\frac{M}{p_{2}}=0 \text { and } \quad \frac{1}{p_{1}} \frac{Q^{-p_{2}} t^{\frac{p_{2}}{p_{1}}}-s}{1-s}-\frac{1}{p_{2}} \frac{t-s}{1-s}=0 .
$$

and so,

$$
t-s=0 \quad \Longrightarrow \quad t=s,
$$

which contradicts Remark 7.4. This finishes the proof.

Note that our change of variables is obviously an open map. Therefore, the interior of the domain of $F$ maps onto the interior of the domain of $G$ and thus $F$ does not attain its maximum in the interior of its domain.

Let us study $F$ on the boundary of its domain.

Case $\alpha=0$. Here everything is obvious, because, independently of $\beta, F(0, \beta)=Q$.

Case $\beta=a$. Here everything is also easy, since the third line of the definition of $w$ (see equation (6.4)) is not involved and, therefore, we have a combination of $(1,1)$ and $\left(v_{-}^{p_{1}}, v_{-}^{p_{2}}\right)$.

Case $\alpha=\frac{\gamma_{-}^{p_{1}}-v_{-}^{p_{1}}}{1-v_{-}^{p_{1}}} a$. This case is already done, since here the first part of $w$ is not involved, and we get a cut-off of the function $u$ from Lemma 7.1.

Case $\beta=1$. This case is more complicated and needs to be studied in detail. Here we have

$$
\left\langle w^{p_{1}}\right\rangle_{J}^{1 / p_{1}}\left\langle w^{p_{2}}\right\rangle_{J}^{-1 / p_{2}}=\left(\frac{x_{1}-\alpha}{1-\alpha}\right)^{1 / p_{1}}\left(\frac{x_{2}-\alpha}{1-\alpha}\right)^{-1 / p_{2}}
$$

where

$$
0 \leqslant \alpha<\frac{\gamma_{-}^{p_{1}}-v_{-}^{p_{1}}}{1-v_{-}^{p_{1}}} a
$$

We define

$$
H\left(x_{1}, \alpha\right)=\left(\frac{x_{1}-\alpha}{1-\alpha}\right)^{1 / p_{1}}\left(\frac{x_{2}-\alpha}{1-\alpha}\right)^{-1 / p_{2}} .
$$

Recall that $x_{2}=Q^{-p_{2}} x_{1}^{p_{2} / p_{1}}$. We need the following observation:

Lemma 7.5. The following is true:

$$
\operatorname{sign}\left(H_{x_{1}}^{\prime}\right)=\operatorname{sign}\left(p_{1}\right)
$$

Supposing for a moment that we have proved this lemma, we show how to finish the proof of Lemma 7.2. We note that $x_{1} \leqslant \gamma_{-}^{p_{1}}$ if $p_{1}>0$ and $x_{1} \geqslant \gamma_{-}^{p_{1}}$ if $p_{1}<0$. Therefore,

$$
H\left(x_{1}, \alpha\right) \leqslant H\left(\gamma_{-}^{p_{1}}, \alpha\right)
$$


We would like to estimate the right-hand side. To this end, assume that $x_{1}=\gamma_{-}^{p_{1}}$ and $x_{2}=Q^{-p_{2}} \gamma_{-}^{p_{2}}$. Let

$$
q(t)= \begin{cases}1, & t \in\left[0, \frac{\gamma_{-}^{p_{1}}-v_{-}^{p_{1}}}{1-v_{-}^{p_{1}}}\right), \\ v_{-}, & t \in\left[\frac{\gamma_{-}^{p_{1}}-v_{-}^{p_{-}}}{1-v_{-}^{p_{1}}}, 1\right] .\end{cases}
$$

Note that $\left\langle q^{p_{1}}\right\rangle=\gamma_{-}^{p_{1}}=x_{1},\left\langle q^{p_{2}}\right\rangle=Q^{-p_{2}} \gamma_{-}^{p_{2}}=x_{2}$ and for every $\alpha \leqslant \frac{\gamma_{-}^{p_{1}}-v_{-}^{p_{1}}}{1-v_{-}^{p_{1}}}$ we have $\left\langle q^{p_{k}}\right\rangle_{[\alpha, 1]}=\frac{x_{k}-\alpha}{1-\alpha}$. Since the whole line segment connecting $(1,1)$ and $\left(v_{-}^{p_{1}}, v_{-}^{p_{2}}\right)$ lies in $\Omega$, we have

$$
\left(\frac{x_{1}-\alpha}{1-\alpha}\right)^{1 / p_{1}}\left(\frac{x_{2}-\alpha}{1-\alpha}\right)^{-1 / p_{2}} \leqslant Q
$$

for every $\alpha \leqslant \frac{\gamma_{-}^{p_{1}}-v_{-}^{p_{1}}}{1-v_{-}^{p_{1}}}$.

However, to estimate $H$ we need to consider $\alpha \leqslant \frac{\gamma_{-}^{p_{1}}-v_{-}^{p_{1}}}{1-v_{-}^{p_{1}}} a$, which is stronger than the previous inequality, since $a \leqslant 1$. Therefore,

$$
H\left(\gamma_{-}^{p_{1}}, \alpha\right) \leqslant Q
$$

There remains to prove Lemma 7.5.

Proof of Lemma 7.5. Recall that

$$
x_{2}=Q^{-p_{2}} x_{1}^{p_{2} / p_{1}} .
$$

Therefore,

$$
\frac{d x_{2}}{d x_{1}}=\frac{p_{2}}{p_{1}} \frac{x_{2}}{x_{1}}
$$

Letting $M=\frac{x_{1}-\alpha}{1-\alpha}$ and $N=\frac{x_{2}-\alpha}{1-\alpha}$, we get:

$$
\begin{aligned}
\frac{\partial H}{\partial x_{1}} & =\frac{1}{p_{1}} M^{1 / p_{1}-1} N^{-1 / p_{2}}-\frac{1}{p_{2}} M^{1 / p_{1}} N^{-1 / p_{2}-1} \frac{p_{2} x_{2}}{p_{1} x_{1}} \\
& =M^{1 / p_{1}-1} N^{-1 / p_{2}-1}\left(\frac{1}{p_{1}} \frac{x_{2}-\alpha}{1-\alpha}-\frac{1}{p_{2}} \frac{x_{1}-\alpha}{1-\alpha} \frac{p_{2}}{p_{1}} \frac{x_{2}}{x_{1}}\right) \\
& =M^{1 / p_{1}-1} N^{-1 / p_{2}-1} \frac{\alpha}{1-\alpha} \frac{1}{x_{1}} \frac{x_{2}-x_{1}}{p_{1}} .
\end{aligned}
$$

All we need to prove now is that $x_{2}-x_{1} \geqslant 0$. Notice that $x_{2} \geqslant x_{1}$ if and only if $Q^{-p_{2}} \geqslant x_{1}^{\left(p_{1}-p_{2}\right) / p_{1}}$, which is true if and only if

$$
p_{1} Q^{\frac{p_{1} p_{2}}{p_{2}-p_{1}}} \geqslant p_{1} x_{1} .
$$

Recall that

$$
Q^{-p_{2}}\left(1-\frac{p_{2}}{p_{1}}\right) \gamma^{p_{2}}=1-\frac{p_{2}}{p_{1}} Q^{-p_{2}} \gamma^{p_{2}-p_{1}}
$$


We have the following chain:

$$
\begin{aligned}
Q^{\frac{p_{1} p_{2}}{p_{2}-p_{1}} \geqslant \gamma_{-}^{p_{1}}} & \Longleftrightarrow Q^{p_{2}} p_{1} \leqslant \gamma_{-}^{p_{2}-p_{1}} p_{1} \Longleftrightarrow p_{1} Q^{-p_{2}} \gamma_{-}^{p_{2}-p_{1}} \geqslant p_{1} \\
& \Longleftrightarrow p_{1} \frac{p_{1}}{p_{2}}\left(1-Q^{-p_{2}}\left(1-\frac{p_{2}}{p_{1}}\right) \gamma_{-}^{p_{2}}\right) \geqslant p_{1} \\
& \Longleftrightarrow p_{1}\left(\frac{p_{1}}{p_{2}}-1\right) \geqslant \frac{p_{1}}{p_{2}}\left(p_{1}-p_{2}\right) Q^{-p_{2}} \gamma_{-}^{p_{2}} \\
& \Longleftrightarrow \frac{p_{1}}{p_{2}} \geqslant \frac{p_{1}}{p_{2}} Q^{-p_{2}} \gamma_{-}^{p_{2}} \Longleftrightarrow p_{1} \frac{Q^{p_{2}}-\gamma_{-}^{p_{2}}}{p_{2}} \geqslant 0 .
\end{aligned}
$$

Since $Q>\gamma_{-}$we get that

$$
Q^{\frac{p_{1} p_{2}}{p_{2}-p_{1}}} \geqslant \gamma_{-}^{p_{1}} \Longleftrightarrow p_{1} \geqslant 0 .
$$

Therefore,

$$
p_{1} Q^{\frac{p_{1} p_{2}}{p_{2}-p_{1}}} \geqslant p_{1} \gamma_{-}^{p_{1}} .
$$

Since we know that $p_{1} \gamma_{-}^{p_{1}} \geqslant p_{1} x_{1}$, this finishes our proof.

\section{Illustration: the $A_{2}$ case and the reverse Hölder property}

This section has two goals. The first one is to write the answer for the Bellman function in the particular case of $p_{1}=1$ and $p_{2}=-1$. This case is interesting because it corresponds to the $A_{2}$ condition, which plays a major role in the theory of singular integral operators. It is also interesting because here we can write an explicit answer in terms of the $A_{2}$-characteristic of the weight, avoiding all implicit functions.

The second goal of this section is to prove the following statement:

Theorem 8.1. Suppose $w \in A_{2}$ and $[w]_{2}=\sup _{I}\langle w\rangle_{I}\left\langle w^{-1}\right\rangle_{I}=Q$. Then there exists a constant $\alpha_{0}>0$, depending only on $Q$, such that for every $\alpha, 0<\alpha<\alpha_{0}$, the following inequality holds:

$$
\left\langle w^{1+\alpha}\right\rangle \leqslant C\langle w\rangle^{1+\alpha}
$$

where $C=C(\alpha)$ is a constant that does not depend on $w$.

We refer one more time to the paper [2], where the opposite question was considered: a reverse Hölder weight self-improves to an $A_{p}$ weight.

We should say that this result is known. It was proved, for example, in [9] with a sharp constant $C$. Our result, compared to Vasyunin's, does not give the sharp constant $C$, but gives the sharp estimate for $\alpha_{0}$. We notice that in the bounds for singular integral operators the sharp dependence of the self-improvement bound $\alpha$ on the characteristic of the weight is the important thing.

Again, we note that we give the proof as an application of our sharp estimate for a distribution function of $A_{2}$ weights. 
Let us start by calculating the function $B$ (see (3.4)) in our particular case. We remind the reader that in our case

$$
B\left(x_{1}, x_{2}\right)=\sup \left\{|\{t: w(t) \geqslant 1\}|:\langle w\rangle=x_{1},\left\langle w^{-1}\right\rangle=x_{2}, w \in A_{2}^{Q}\right\} .
$$

We start with calculating the constants $\gamma_{ \pm}$. We have the equation

$$
Q(1+1) \frac{1}{\gamma}=1+Q \frac{1}{\gamma^{2}}
$$

which has two solutions

$$
\gamma_{+}=Q+\sqrt{Q^{2}-Q} \text { and } \gamma_{-}=Q-\sqrt{Q^{2}-Q} .
$$

Therefore,

$$
v_{-}=\frac{\gamma_{-}}{\gamma_{+}}=\frac{Q-\sqrt{Q^{2}-Q}}{Q+\sqrt{Q^{2}-Q}} .
$$

We know that in $\Omega_{I}$ our function $B$ equals 1 . Let us calculate the numbers $a, b$ and $c$ for $\Omega_{\mathrm{II}}$. We have

$$
\begin{aligned}
1-v_{-} & =\frac{2 \sqrt{Q^{2}-Q}}{Q+\sqrt{Q^{2}-Q}}, \\
v_{-}^{-1}-1 & =\frac{2 \sqrt{Q^{2}-Q}}{Q-\sqrt{Q^{2}-Q}}, \\
v_{-}-v_{-}^{-1} & =-4 \sqrt{Q^{2}-Q} .
\end{aligned}
$$

Furthermore,

$$
\begin{aligned}
& a=\frac{v_{-}}{\left(1-v_{-}\right)\left(v_{-}-v_{-}^{-1}\right)}=-\frac{Q-\sqrt{Q^{2}-Q}}{8\left(Q^{2}-Q\right)}, \\
& b=\frac{v_{-}^{-1}}{\left(v_{-}^{-1}-1\right)\left(v_{-}-v_{-}^{-1}\right)}=-\frac{Q+\sqrt{Q^{2}-Q}}{8\left(Q^{2}-Q\right)}, \\
& c=1-\frac{1}{\left(v_{-}-1\right)\left(v_{-}^{-1}-1\right)}=1+\frac{1}{4(Q-1)} .
\end{aligned}
$$

We proceed to the domain $\Omega_{\mathrm{III}}$. Let us find the parameter $v$ in terms of $x_{1}$ and $x_{2}$. We have the equation

$$
\frac{1}{v}\left(1-x_{1}\right)-v\left(1-x_{2}\right)=x_{2}-x_{1} .
$$

This is a quadratic equation and its roots are

$$
v=1 \quad \text { and } \quad v=\frac{1-x_{1}}{x_{2}-1} .
$$


Therefore, in $\Omega_{\mathrm{III}}$ we have

$$
B(x)=\frac{x_{1} x_{2}-1}{x_{1}+x_{2}-2} .
$$

In the domain $\Omega_{\mathrm{IV}}$ we need to do more work. First of all, we should again find our $v$. Our equation (again quadratic) is the following:

$$
x_{2}=-Q \gamma_{+}^{-2} v^{-2}\left(x_{1}-v\right)+\frac{1}{v}
$$

which reduces to

$$
x_{2} v^{2}-v\left(1+\frac{Q}{\gamma_{+}^{2}}\right)+\frac{Q}{\gamma_{+}^{2}} x_{1}=0 .
$$

The roots of this equation are

$$
v=\frac{\left(1+Q / \gamma_{+}^{2}\right) \pm \sqrt{\left(1+Q / \gamma_{+}^{2}\right)^{2}-4\left(Q / \gamma_{+}^{2}\right) x_{1} x_{2}}}{2 x_{2}} .
$$

We should take the bigger value of $v$. This is obvious from the following picture. The interested reader can check this algebraically.

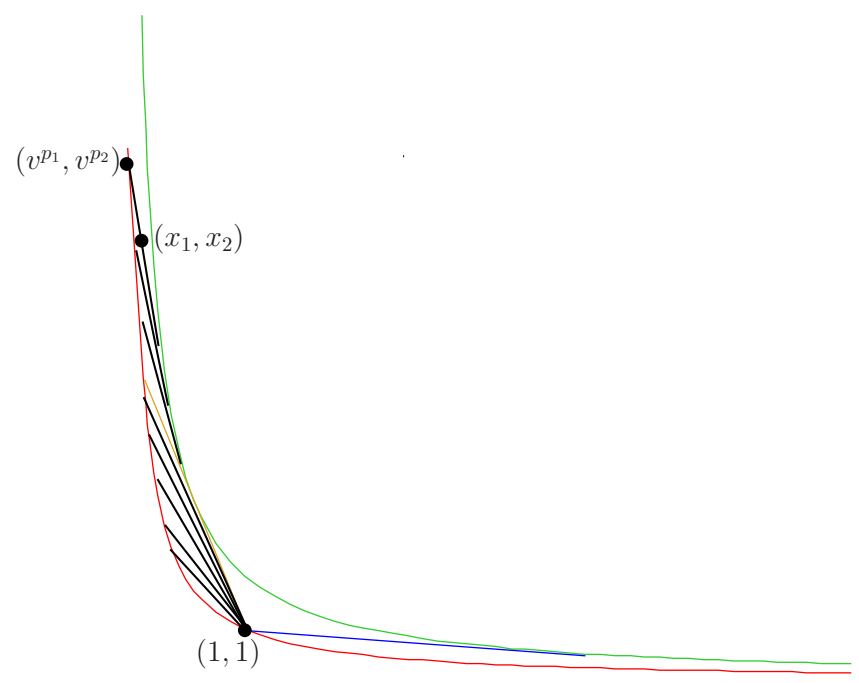

Figure 7: The foliation for the case $p_{1}=1$ and $p_{2}=-1$.

So, we put

$$
v=\frac{\left(1+Q / \gamma_{+}^{2}\right)+\sqrt{\left(1+Q / \gamma_{+}^{2}\right)^{2}-4\left(Q / \gamma_{+}^{2}\right) x_{1} x_{2}}}{2 x_{2}} .
$$

Using

$$
\frac{Q}{\gamma_{+}^{2}}=Q \frac{\gamma_{-}}{\gamma_{+}} \frac{1}{\gamma_{-} \gamma_{+}}=v_{-}
$$


we get

$$
v=\frac{1+v_{-}+\sqrt{\left(1+v_{-}\right)^{2}-4 v_{-} x_{1} x_{2}}}{2 x_{2}} .
$$

Recall that

$$
A=\frac{Q}{\gamma_{+}^{2}}=v_{-} .
$$

Therefore, we get a simple answer for $B$ in $\Omega_{\mathrm{IV}}$ :

$$
\begin{aligned}
B(x) & =\frac{1}{1-v_{-}} \frac{v_{-}^{-\frac{2 v_{-}}{1-v_{-}}}}{1-v_{-}} v^{\frac{2}{1-v_{-}}}\left(x_{2}+\frac{x_{1}}{v^{2}}-\frac{2}{v}\right) \\
& =\frac{1}{1-v_{-}} \frac{v_{-}^{-\frac{2 v_{-}}{1-v_{-}}}}{1-v_{-}} v^{\frac{2}{1-v_{-}}-2}\left(v^{2} x_{2}+x_{1}-2 v\right) .
\end{aligned}
$$

Since

$$
v^{2} x_{2}=v-v_{-}\left(x_{1}-v\right),
$$

we get

$$
B(x)=\frac{v_{-}^{-\frac{2 v_{-}}{1-v_{-}}}}{1-v_{-}} v^{\frac{2 v_{-}}{1-v_{-}}}\left(x_{1}-v\right)
$$

Finally,

$$
x_{1}-v=\frac{2 x_{1} x_{2}-\left(1+v_{-}+\sqrt{\left(1+v_{-}\right)^{2}-4 v_{-} x_{1} x_{2}}\right)}{2 x_{2}} .
$$

We know that $x_{1} x_{2} \leqslant Q$, so

$$
x_{1}-v \leqslant \frac{2 Q-1-v_{-}-\sqrt{\left(1+v_{-}\right)^{2}-4 Q v_{-}}}{2 x_{2}} .
$$

The right-hand side is equal to $\sqrt{Q^{2}-Q} / x_{2}$, so we get

$$
x_{1}-v \leqslant \frac{\sqrt{Q^{2}-Q}}{x_{2}} \text {. }
$$

Note that this estimate is in some sense sharp. We cannot guarantee that if our $w$ has the $A_{2}$-characteristic equal to $Q$ then it is attained on the initial interval. But there are a lot of functions for which this is the case, for example, the one from Section 6.

We get the following estimate for $B(x)$, when $x \in \Omega_{\mathrm{IV}}$ :

$$
B(x) \leqslant \frac{v_{-}^{-\frac{2 v_{-}}{1-v_{-}}}}{1-v_{-}} v^{\frac{2 v_{-}}{1-v_{-}}} \frac{\sqrt{Q^{2}-Q}}{x_{2}} .
$$


Moreover, since $x_{1} x_{2} \in[1, Q]$, and using (8.7), we get

$$
v \asymp \frac{1}{x_{2}} \text {. }
$$

Therefore,

$$
B(x) \leqslant C(Q) x_{2}^{-\frac{Q}{\sqrt{Q^{2}-Q}}} .
$$

We also note that if $x_{1} x_{2}=Q$ then

$$
C(Q)=\frac{\gamma_{+}^{\frac{2 v_{-}}{1-v_{-}}}}{1-v_{-}} \sqrt{Q^{2}-Q} .
$$

Altogether we have the following compound formula for $B$ :

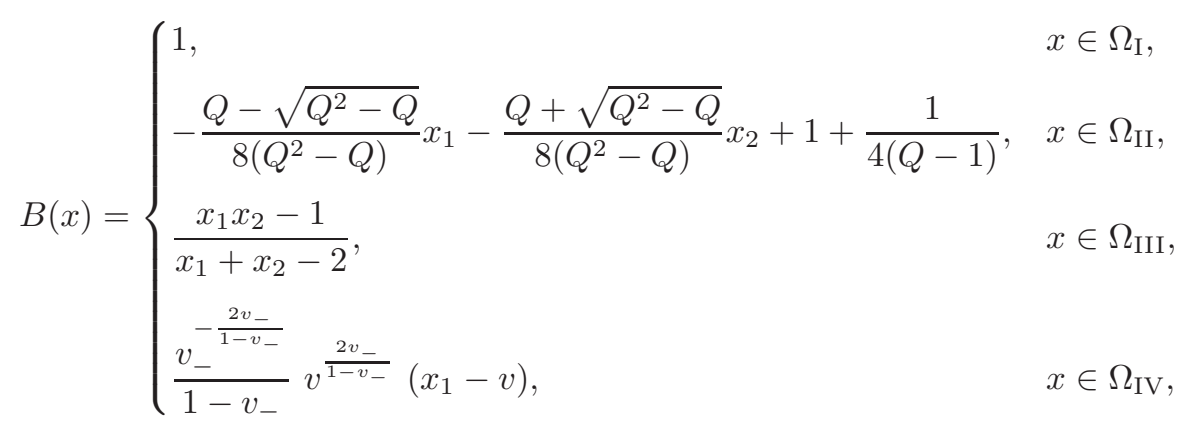

as well as the corresponding estimate:

$$
B(x) \leqslant \begin{cases}1, & x \in \Omega_{\mathrm{I}} \cup \Omega_{\mathrm{II}}, \\ \frac{Q-1}{x_{1}+x_{2}-2}, & x \in \Omega_{\mathrm{III}}, \\ C(Q) x_{2}^{-\frac{Q}{\sqrt{Q^{2}-Q}}}, & x \in \Omega_{\mathrm{IV}} .\end{cases}
$$

Now we proceed to the reverse Hölder property, i.e., Theorem 8.1. Without loss of generality we consider only the case when the function $w$ satisfies $\langle w\rangle\left\langle w^{-1}\right\rangle=Q$. This simplifies things a little since then the point $\left(\langle t w\rangle,\left\langle t^{-1} w^{-1}\right\rangle\right)$ is on the curve $\Gamma_{Q}$ and thus never in $\Omega_{\mathrm{III}}$.

Next, we use that

$$
\left\langle w^{1+\alpha}\right\rangle=(1+\alpha) \int_{0}^{\infty} s^{\alpha} F_{w}(s) d s,
$$

where

$$
F_{w}(s)=|\{t: w(t) \geqslant s\}|
$$

We know that

$$
F_{w}(s) \leqslant B\left(x_{1}, x_{2} ; s\right)=B\left(\frac{x_{1}}{s}, x_{2} s\right)
$$


thus

$$
\left\langle w^{1+\alpha}\right\rangle \leqslant(1+\alpha) \int_{0}^{\infty} s^{\alpha} B\left(\frac{x_{1}}{s}, x_{2} s\right) d s .
$$

We consider the point $S=\left(x_{1} / s, x_{1} s\right)$. Note that

$$
S \in \begin{cases}\Omega_{\mathrm{I}}, & s<x_{1} / \gamma_{+}, \\ \Omega_{\mathrm{II}}, & s \in\left(x_{1} / \gamma_{+}, x_{1} / \gamma_{-}\right), \\ \Omega_{\mathrm{IV}}, & s>x_{1} / \gamma_{-} .\end{cases}
$$

Thus,

$$
\begin{aligned}
\left\langle w^{1+\alpha}\right\rangle & \leqslant(1+\alpha) \int_{0}^{\infty} s^{\alpha} B\left(\frac{x_{1}}{s}, x_{2} s\right) d s \leqslant \\
& \leqslant C\left(\int_{0}^{x_{1} / \gamma_{-}} s^{\alpha} d s+\int_{x_{1} / \gamma_{-}}^{\infty} s^{\alpha} x_{2}^{-\frac{Q}{\sqrt{Q^{2}-Q}}} s^{-\frac{Q}{\sqrt{Q^{2}-Q}}} d s\right) .
\end{aligned}
$$

Note that the right-hand side gives us an estimate of the form $C x_{1}^{1+\alpha}=C\langle w\rangle^{1+\alpha}$ as long as the second integral converges at $\infty$. It does when

$$
\alpha-\frac{Q}{\sqrt{Q^{2}-Q}}<-1,
$$

or, equivalently,

$$
\alpha<\alpha_{0} \stackrel{\text { def }}{=} \sqrt{\frac{Q}{Q-1}}-1 .
$$

This finishes our proof.

\section{Some final remarks}

In this section we comment on some cases that we did not consider.

First of all, we did not consider the cases $p_{k}=0, \pm \infty$. However, in these cases our method works in the same way. In the case $p=0$ the expression $\left\langle w^{p}\right\rangle_{J}^{1 / p}$ has to be replaced by $\exp \langle\log w\rangle_{J}$. It has to be replaced by $\sup _{J} w$ in the case $p=+\infty$, and by $\inf _{J} w$ in the case $p=-\infty$. The answer for $\mathcal{B}$ in these cases will be, for example, obtained by passing to the limit when $p_{k} \rightarrow 0, \pm \infty$. Another way to get the answer in these cases is to find a system of differential equations, similar to our Monge-Ampère equation. For details the reader can see [9].

We also note that for the $A_{\infty}$ case, i.e., when $p_{1}=1$ and $p_{2}=0$, one can get an answer solving the Monge-Ampère equation. The correct variables will be $x_{1}=\langle w\rangle$ and $x_{2}=\langle\log w\rangle$ with the relation

$$
x_{1} \exp \left(-x_{2}\right) \in[1, Q]
$$


With the same splitting of the domain as before, the answer is the following:

$$
B(x)= \begin{cases}1, & x \in \Omega_{\mathrm{I}}, \\ -\frac{v_{-}}{\left(v_{-}-1\right)^{2}} x_{1}+\frac{1}{\log \left(v_{-}\right)} \frac{1}{v_{-}-1} x_{2}+\left(1+\frac{v_{-}}{\left(v_{-}-1\right)^{2}}\right), & x \in \Omega_{\mathrm{II}}, \\ \frac{x_{1}-v}{1-v}, & x \in \Omega_{\mathrm{III}}, \\ \frac{\gamma_{+}}{\gamma_{+}-1} \frac{1}{1-v_{-}} \cdot\left(x_{1}-x_{2} v-v(1-\log (v))\right), & x \in \Omega_{\mathrm{IV}} .\end{cases}
$$

where the function $v$ is defined by an implicit formula:

$$
\begin{cases}x_{2}(1-v)=\left(1-x_{1}\right) \log (v), & x \in \Omega_{\mathrm{III}}, \\ v x_{2}=\frac{1}{\gamma_{+}}\left(x_{1}-v\right)+v \log (v), & x \in \Omega_{\mathrm{IV}} .\end{cases}
$$

We can further modify our setting by considering another Bellman function where we calculate

$$
\sup (|\{w>1\}|, \ldots) .
$$

The answer will be the same at every point except $(1,1)$, where our new function will be zero. Also we will not have an extremal function for every point; instead, we need to build extremal sequences.

Moreover, since

$$
|\{w \leqslant 1\}|=1-|\{w>1\}|,
$$

we get that

$$
\sup (|\{w \leqslant 1\}|, \ldots)=1-\inf (|\{w>1\}|, \ldots) .
$$

Using our technique, one can easily calculate the right-hand side. The function for inf can be calculated in the same way as $\mathcal{B}$ with one difference: it must be convex rather than concave.

\section{References}

[1] Burkholder, D.: Sharp inequalities for martingales and stochastic integrals. Astérisque 157-158 (1988), 75-94.

[2] Dindoš, M. And Wall, T.: The sharp $A_{p}$ constant for weights in a reverse-Hölder class. Rev. Mat. Iberoam. 25 (2009), no. 2, 559-594.

[3] Hytönen, T. And Pérez, C.: Sharp weighted bounds involving $A_{\infty}$. J. Funct. Anal. 263 (2012), no. 12, 3883-3899.

[4] Nazarov, F., Treil, S. And Volberg, A.: The Bellman function and two-weight inequality for Haar multipliers. J. Amer. Math. Soc. 12 (1999), no. 4, 909-928.

[5] PÉrez, C.: The growth of the $A_{p}$ constant on classical estimates. Rev. Un. Mat. Argentina 50 (2009), no. 2, 119-135.

[6] Slavin, L. And Vasyunin, V.: Sharp results in the integral-form John-Nirenberg inequality. Trans. Amer. Math. Soc. 363 (2011), no. 8, 4135-4169. 
[7] Slavin, L. And Vasyunin, V.: Sharp $L^{p}$ estimates on BMO. To appear in Indiana U. Math. J.

[8] Vasyunin, V.: The exact constant in the inverse Hölder inequality for Muckenhoupt weights. Translation in St. Petersburg Math. J. 15 (2004), no. 1, 49-79.

[9] Vasyunin, V.: Mutual estimates for $L^{p}$-norms and the Bellman function. Traslation in J. Math. Sci. (N. Y.) 156 (2009), no. 5, 766-798.

[10] Vasyunin, V.: Sharp constants in the classical weak form of the John-Nirenberg inequality. Preprint of the Petersburg Department of Steklov Institute of Mathematics, http://www.pdmi.ras.ru/preprint/2011/eng-2011.html, 2011.

[11] Vasyunin, V. And Volberg, A.: Monge-Ampère equation and Bellman optimization of Carleson embedding theorems. In Linear and complex analysis 195-238. Amer. Math. Soc. Transl. Ser. 2, 226, Amer. Math. Soc., Providence, RI, 2009.

Received May 24, 2011; revised February 2, 2012.

Alexander Reznikov: Department of Mathematics, Michigan State University, East Lansing, MI 48824, USA.

E-mail: rezniko2@msu.edu 